\title{
Macroscopic Irreversibility and Decay to Kinetic Equilibrium of the 1-Body PDF for Finite Hard-Sphere Systems
}

\author{
Massimo Tessarotto $\mathbb{D}^{1,2}$ and Claudio Cremaschini $\mathbb{D}^{3}$ \\ ${ }^{1}$ Department of Mathematics and Geosciences, University of Trieste, Via Valerio 12, 34127 Trieste, Italy \\ ${ }^{2}$ Institute of Physics, Faculty of Philosophy and Science, Silesian University in Opava, Bezručovo nám. 13, 74601 Opava, Czech Republic \\ ${ }^{3}$ Institute of Physics and Research Center for Theoretical Physics and Astrophysics, Faculty of Philosophy and Science, \\ Silesian University in Opava, Bezručovo nám. 13, 74601 Opava, Czech Republic
}

Correspondence should be addressed to Massimo Tessarotto; maxtextss@gmail.com

Received 29 August 2018; Accepted 5 November 2018; Published 2 December 2018

Academic Editor: Yannis Dimakopoulos

Copyright ( 2018 Massimo Tessarotto and Claudio Cremaschini. This is an open access article distributed under the Creative Commons Attribution License, which permits unrestricted use, distribution, and reproduction in any medium, provided the original work is properly cited.

\begin{abstract}
The conditions for the occurrence of the so-called macroscopic irreversibility property and the related phenomenon of decay to kinetic equilibrium which may characterize the 1-body probability density function (PDF) associated with hard-sphere systems are investigated. The problem is set in the framework of the axiomatic "ab initio" theory of classical statistical mechanics developed recently and the related establishment of an exact kinetic equation realized by the Master equation for the same kinetic PDF. As shown in the paper the task involves the introduction of a suitable functional of the 1-body PDF, identified here with the Master kinetic information. It is then proved that, provided the same PDF is prescribed in terms of suitably smooth, i.e., stochastic, solution of the Master kinetic equation, the two properties indicated above are indeed realized.
\end{abstract}

\section{Introduction}

The axiomatic theory of classical statistical mechanics (CSM) recently proposed in a series of papers (see [1-4]) and referred to as $a b$ initio theory of CSM provides a self-consistent pathway to the kinetic theory of hard-sphere systems, as well as in principle also point particles subject to finite-range interactions [5]. Its theoretical basis and conditions of validity are indeed founded on a unique physical realization of the axioms which are set at the foundations of CSM [1-3], a fact which permits the treatment of phase-space and kinetic probability density functions (PDF) which are realized by either stochastic (i.e., ordinary) functions or distributions such as the $N$-body Dirac delta (or certainty function [6]). This feature is physically based being due to the prescription of the collision boundary conditions ( $\mathrm{CBC},[2])$, i.e., the relationship occurring at collision events between incoming and outgoing multibody probability density functions PDF. The choice of the appropriate $\mathrm{CBC}$ indicated in [2], denoted as modified collision boundary condition (MCBC), is actually of crucial importance and departs from the customary realization/interpretation (of the same axioms) originally adopted in Boltzmann [7], Enskog [8, 9], and Grad [10] kinetic approaches (for a review of Grad's kinetic theory based on CSM see also Cercignani [11, 12]). The same choice implies, in fact, a number of theoretical and physically relevant consequences. In particular, it follows that the new theory

(i) unlike Enskog theory [3] applies also to finite $\mathrm{N}$-body hard-sphere systems $S_{N}$, namely, systems formed by $N$ like smooth hard-spheres of diameter $\sigma$ and mass $m$, in which the parameters $(N, \sigma, m)$ remain all constant and finite [3]. On the other hand, the same particles are assumed as usual: (A) subject to instantaneous (unary, binary, and multiple) elastic collisions which leave unchanged the particles angular momenta and (B) immersed in a stationary bounded domain $\Omega$ of the Euclidean space $\mathbb{R}^{3}$ with finite canonical measure;

(ii) has led to the discovery [3] of an exact kinetic equation holding globally in time [13] (i.e., for all $t \in I \equiv$ $\mathbb{R})$ for these systems and denoted as Master kinetic 
equation (recalled in Appendix A). In other words the Master equation is nonasymptotic in character with respect to the parameters the (finite) parameters $(N, \sigma, m)$. In addition the same equation holds under suitable maximal entropy conditions for the statistical treatment of the so-called Boltzmann-Sinai classical dynamical system (CDS), which implies that initial (binary or multibody) phase-space statistical correlations are assumed identically vanishing, while at the same time only suitable uniquely prescribed configuration-space correlations can arise. As such the equation generalizes and extends the validity of the Boltzmann and Enskog kinetic equations and notably applies to arbitrary 1-body PDFs which can be realized either in terms of stochastic functions or distributions;

(iii) is time-reversal invariant [4], namely, the Master kinetic equation is time-reversal $(\mathscr{T} \mathscr{R}-)$ symmetric. In other words, the same equation is invariant with respect to the $\mathscr{T} \mathscr{R}$-transformation

$$
\begin{aligned}
& \tau \equiv t-t_{o} \longrightarrow \tau^{\prime} \equiv t^{\prime}-t_{o}=-t+t_{o} \equiv-\tau, \\
& \mathbf{r}_{1} \longrightarrow \mathbf{r}_{1}^{\prime}=\mathbf{r}_{1}, \\
& \mathbf{v}_{1} \longrightarrow \mathbf{v}_{1}^{\prime}=-\mathbf{v}_{1} .
\end{aligned}
$$

Thus, representing the absolute time as $t=\tau+t_{o}$, with $t_{o}$ being a prescribed (arbitrary) initial time, it follows that the $\mathscr{T} \mathscr{R}$-transformation leaves invariant the initial time $t_{o}$ and the instantaneous position $\mathbf{r}_{1}=$ $\mathbf{r}_{1}\left(t \equiv t_{o}+\tau\right)$ of an arbitrary particle, while reversing the signature (i.e., versus) of its velocity $\mathbf{v}_{1}=\mathbf{v}_{1}(t \equiv$ $\left.t_{o}+\tau\right)$. Accordingly, thanks to $\mathscr{T} \mathscr{R}$-symmetry the two initial-value problems associated with the Master kinetic equation in the two cases are related in such a way that, respectively, the initial 1-body PDF at time $t_{o}, \rho_{1}^{(N)}\left(\mathbf{x}_{1}, t_{o}\right) \equiv \rho_{1(o)}^{(N)}\left(\mathbf{r}_{1}, \mathbf{v}_{1}\right)$, and the corresponding time-evolved PDF $\rho_{1}^{(N)}\left(\mathbf{x}_{1}, t\right)$ are carried into the $\mathscr{T} \mathscr{R}$-transformed 1-body PDFs $\rho_{1(o) \mathscr{T} \mathscr{R}}^{(N)}\left(\mathbf{r}_{1}^{\prime}, \mathbf{v}_{1}^{\prime}\right)$ and $\rho_{1 \mathscr{T} \mathscr{R}}^{(N)}\left(\mathbf{r}_{1}^{\prime}, \mathbf{v}_{1}^{\prime}, t^{\prime}\right)$, respectively, prescribed according to the law

$$
\begin{gathered}
\rho_{1(o)}^{(N)}\left(\mathbf{r}_{1}, \mathbf{v}_{1}\right) \\
\rho_{1}^{(N)}\left(\mathbf{x}_{1}, t\right) \equiv \rho_{1}^{(N)}\left(\mathbf{r}_{1}, \mathbf{v}_{1}, t_{o}+\tau\right) \\
\downarrow \\
\rho_{1(o) \mathscr{T} \mathscr{R}}^{(N)}\left(\mathbf{r}_{1}^{\prime}, \mathbf{v}_{1}^{\prime}\right) \equiv \rho_{1(o)}^{(N)}\left(\mathbf{r}_{1},-\mathbf{v}_{1}\right) \\
\rho_{1 \mathscr{T} \mathscr{R}}^{(N)}\left(\mathbf{r}_{1}^{\prime}, \mathbf{v}_{1}^{\prime}, t^{\prime}\right) \equiv \rho_{1}^{(N)}\left(\mathbf{r}_{1},-\mathbf{v}_{1}, t_{o}-\tau\right) ;
\end{gathered}
$$

(iv) conserves the corresponding Boltzmann-Shannon (BS) statistical entropy [4]. This is identified with the phase-space moment

$$
S\left(\rho_{1}^{(N)}(t)\right)-\int_{\Gamma_{1}} d \mathbf{x}_{1} \rho_{1}^{(N)}\left(\mathbf{x}_{1}, t\right) \ln \frac{\rho_{1}^{(N)}\left(\mathbf{x}_{1}, t\right)}{A_{1}}
$$

with $\rho_{1}^{(N)}(t) \equiv \rho_{1}^{(N)}\left(\mathbf{x}_{1}, t\right)$ being an arbitrary stochastic PDF solution of the Master kinetic equation and $A_{1}$ an arbitrary positive constant such that the initial PDF

$$
\rho_{1}^{(N)}\left(t_{o}\right) \equiv \rho_{1}^{(N)}\left(\mathbf{x}_{1}, t_{o}\right)=\rho_{10}^{(N)}\left(\mathbf{x}_{1}\right)
$$

is such that the corresponding BS functional $S\left(\rho_{1}^{(N)}\left(t_{o}\right)\right)$ is defined. As a consequence it follows that an arbitrary smooth solution of the Master kinetic equation satisfies the constant $\mathrm{H}$-theorem

$$
\frac{\partial}{\partial t} S\left(\rho_{1}^{(N)}(t)\right)=0
$$

for all $t \in I \equiv \mathbb{R}$ (see again related discussion in [4]).

Based on the ab initio theory of CSM, in this paper the problem is posed of the existence of two phenomena which are expected to characterize the statistical description of finite $N$-body hard-sphere systems and therefore should lay at the very foundation of CSM and kinetic theory. These are related to the physical conditions for the possible occurrence of the so-called property of macroscopic irreversibility (PMI) and the consequent one represented by the decay to kinetic equilibrium (DKE) which characterize the 1-body (kinetic) PDF in these $N$-body systems, i.e., when 1 -body-factorized initial conditions are considered for the $N$-body Liouville equation [3]. The conjecture is that-in some sense in analogy with the ubiquitous character of the ergodicity property which characterizes hard-sphere systems and hence the $S_{N}-$ CDS $[14,15]$ - the occurrence of such phenomena should be independent of the number $N$ of constituent particles of the system and therefore apply to actual physical systems for which the parameters $(N, \sigma, m)$ are obviously all finite.

1.1. Motivations and Background. Both properties indicated above concern the statistical behavior of an ensemble $S_{N}$ of like particles which are advanced in time by a suitable $N$-body classical dynamical system, identified here with the $S_{N}-$ CDS. Specifically they arise in the context of the kinetic description of the same CDS, i.e., in terms of the corresponding 1-body (kinetic) probability density function (PDF) $\rho_{1}^{(N)}(t) \equiv \rho_{1}^{(N)}\left(\mathbf{x}_{1}, t\right)$. The latter is required to belong to the functional class $\left\{\rho_{1}^{(N)}\left(\mathbf{x}_{1}, t\right)\right\}$ of suitably smooth and strictly positive ordinary functions which are particular solutions of the relevant kinetic equation.

In fact, PMI should be realized by means of a suitable, but still possibly nonunique, functional which should be globally defined in the future (i.e., for all times $t \geq t_{o}$, being $t_{o}$ the initial time) bounded and nonnegative, and therefore to be identified with the notion of information measure. Most importantly, however, the same functional, to be referred to here as Master kinetic information (MKI), should also exhibit a continuously differentiable and monotonic, i.e., in particular decreasing, time-dependence.

Regarding, instead, the second property of DKE this concerns the asymptotic behavior of the 1-body $\operatorname{PDF} \rho_{1}^{(N)}(t) \equiv$ $\rho_{1}^{(N)}\left(\mathbf{x}_{1}, t\right)$ which, accordingly, should be globally defined and 
decay for $t \longrightarrow+\infty$ to a stationary and spatially uniform Maxwellian PDF

$$
\rho_{1 M}^{(N)}\left(\mathbf{v}_{1}\right)=\frac{n_{o}}{\pi^{3 / 2}\left(2 T_{o} / m\right)^{3 / 2}} \exp \left\{-\frac{m\left(\mathbf{v}_{1}-\mathbf{v}_{o}\right)^{2}}{2 T_{o}}\right\},
$$

where $\left\{n_{o}>0, T_{o}>0, \mathbf{V}_{o}\right\}$ are constant fluid fields.

Both PMI and DKE correspond to physical phenomena which are actually expected to arise in disparate classical $N$-body systems. The clue for their realization is represented by the ubiquitous occurrence of kinetic equilibria and consequently, in principle, also of the corresponding possible manifestation of macroscopic irreversibility and decay processes. Examples of the former ones are in principle easy to be found, ranging from neutral fluids [16] to collisional/collisionless and nonrelativistic/relativistic gases and plasmas [17-19].

However, the most notable example is perhaps provided by dilute hard-sphere systems ("gases") characterized by a large number of particles $(N \equiv 1 / \varepsilon \gg 1)$ and a small (i.e., infinitesimal) diameter $\sigma \sim O\left(\varepsilon^{1 / 2}\right)$ of the same hard-spheres, for which the Boltzmann equation applies. Indeed the Boltzmann equation is actually specialized to the treatment of dilute hard-sphere systems in the Boltzmann-Grad limit discussed in the Lanford theorem [20-22] (for a detailed discussion of the topics in the context of the ab initio theory see also [4]). In such a case the 1-body PDF can be formally obtained by introducing the Boltzmann-Grad limit operator [4]

$$
\mathscr{L}_{B G} \equiv \lim _{\substack{N \equiv 1 / \varepsilon \longrightarrow \infty \\ \sigma \sim O\left(\varepsilon^{1 / 2}\right)}}
$$

whereby the limit function $\rho_{1}\left(\mathbf{x}_{1}, t\right)$ is denoted

$$
\rho_{1}\left(\mathbf{x}_{1}, t\right)=\mathscr{L}_{B G} \rho_{1}^{(N)}\left(\mathbf{x}_{1}, t\right)
$$

and $\rho_{1}\left(\mathbf{x}_{1}, t\right)$ identifies a particular solution of the Boltzmann kinetic equation.

Historically, the property of irreversibility indicated above is known to be related to the Carnot's second Law of Classical Thermodynamics. More precisely, it is related to the first-principle-proof originally attempted by Ludwig Boltzmann in 1872 [7]. Actually it is generally agreed that both phenomena lie at the very heart of Boltzmann and Grad kinetic theories $[7,10]$ and the related original construction of the Boltzmann kinetic equation (1872). In particular, the goal set by Boltzmann himself in his 1872 paper was the proof of Carnot's Law providing at the same time also a possible identification of thermodynamic entropy. This was achieved in terms of what is nowadays known as Boltzmann-Shannon (BS) statistical entropy, which is identified with the phasespace moment

$$
\begin{aligned}
M_{X_{E}}\left(\rho_{1}(t)\right) & \equiv \int_{\Gamma_{1}} d \mathbf{x}_{1} \rho_{1}\left(\mathbf{x}_{1}, t\right) X_{E}\left(\mathbf{x}_{1}, t\right) \\
& =-\int_{\Gamma_{1}} d \mathbf{x}_{1} \rho_{1}\left(\mathbf{x}_{1}, t\right) \ln \frac{\rho_{1}\left(\mathbf{x}_{1}, t\right)}{A_{1}} \\
& \equiv S\left(\rho_{1}(t)\right) .
\end{aligned}
$$

Here $X_{E}\left(\mathbf{x}_{1}, t\right) \equiv-\ln \left(\rho_{1}\left(\mathbf{x}_{1}, t\right) / A_{1}\right), \rho_{1}\left(\mathbf{x}_{1}, t\right)$, and $A_{1}$ denote, respectively, the $B S$ entropy density, an arbitrary particular solution of the Boltzmann equation for which the same phase-space integral exists and an arbitrary positive constant. In fact, according to the Boltzmann H-theorem [7] the same functional should satisfy the entropic inequality

$$
\frac{\partial}{\partial t} S\left(\rho_{1}(t)\right) \geq 0
$$

while, furthermore, the entropic equality condition

$$
\begin{aligned}
\frac{\partial}{\partial t} S\left(\rho_{1}(t)\right) & =0 \Longleftrightarrow \\
\rho_{1}\left(\mathbf{x}_{1}, t\right) & =\rho_{1 M}^{(N)}\left(\mathbf{v}_{1}\right)
\end{aligned}
$$

should hold. The latter equation implies therefore that, provided $\rho_{1}(t)$ and $S\left(\rho_{1}(t)\right)$ exist globally [23], then necessarily $\lim _{t \rightarrow+\infty} \rho_{1}\left(\mathbf{x}_{1}, t\right)=\rho_{1 M}\left(\mathbf{v}_{1}\right)$, with $\rho_{1 M}\left(\mathbf{v}_{1}\right)$ denoting the stationary and spatially uniform Maxwellian PDF (6).

In this reference, however, the question arises of the precise characterization of the concept of irreversibility, i.e., whether it should be regarded as a purely macroscopic phenomenon ("macroscopic irreversibility"), i.e., affecting only the BS entropy $S\left(\rho_{1}(t)\right)$ through the Boltzmann H-theorem indicated above, or microscopic in the sense that the same Boltzmann equation should be considered as irreversible ("microscopic irreversibility"). Thus, in principle, in the second case the further issue emerges of the possible physical origin of microscopic irreversibility in special reference to the Lanford's derivation of the Boltzmann equation and subsequent related comments discussed respectively by Uffink and Valente and Ardourel in [24, 25] (see also Drory [26]).

However, as shown in [4], the Boltzmann equation is actually $\mathscr{T} \mathscr{R}$-symmetric. Such a conclusion is of basic importance since it overcomes the so-called Loschmidt paradox, i.e., the objection raised by Loschmidt in 1876 [27] regarding the original Boltzmann formulation of his namesake kinetic equation and $\mathrm{H}$-theorem [7]. In fact, Loschmidt claimed that the Boltzmann $\mathrm{H}$-theorem inequality should change sign under time-reversal and thus violate the microscopic time-reversibility of the underlying hard-sphere classical dynamical system. In his long-pondered reply given in 1896 [28] Boltzmann himself introduced what was later referred to as the modified form of the Boltzmann $\mathrm{H}$-theorem [29].

The key implication is therefore that, in contrast to Boltzmann's own statement and the traditional subsequent mainstream literature interpretation (see, for example, by Cercignani, Lebowitz in $[30,31]$ and more recently the review given by Gallavotti [32]), the Boltzmann $\mathrm{H}$ theorem indicated-together with the modified form indicated above-cannot be interpreted as an intrinsic irreversibility property occurring at the microscopic level, namely, holding for the Boltzmann equation itself. On the contrary, consistent with the physical interpretation of the Loschmidt paradox provided in [4], this must be regarded only as property of macroscopic irreversibility (or PMI) of the 1 -body PDF solution of the Boltzmann equation. In other 
words, the Boltzmann inequality (10) necessarily holds independent of the orientation of the time axis (arrow of time) and therefore cannot represent a true (i.e., microscopic) property which as such should uniquely determine the arrow of time.

Nevertheless, the possible realization of either PMI or DKE is more subtle. In fact they actually depend in a critical way on the prescription of the functional class $\left\{\rho^{(N)}\left(\mathbf{x}_{1}, t\right)\right\}$, so that their occurrence is actually nonmandatory. Indeed, both cannot occur-in principle also for Boltzmann and Grad kinetic theories - if the $N$-body probability density function $\rho^{(N)}(\mathbf{x}, t)$ is identified with the deterministic $N$-body PDF [1], namely, the $N$-body phase-space Dirac delta. This is defined as $\delta(\mathbf{x}-\mathbf{x}(t)) \equiv \prod_{1=1, N} \delta\left(\mathbf{x}_{i}-\mathbf{x}_{i}(t)\right)$, with $\mathbf{x} \equiv$ $\left\{\mathbf{x}_{1}, \ldots, \mathbf{x}_{N}\right\}$ denoting the state of the $N$-body system and $\mathbf{x}(t) \equiv\left\{\mathbf{x}_{1}(t), \ldots, \mathbf{x}_{N}(t)\right\}$ is the image of an arbitrary initial state $\mathbf{x}\left(t_{o}\right) \equiv \mathbf{x}_{o}$ generated by the same $N$-body CDS. That such a PDF necessarily must realize an admissible particular solution of the $\mathrm{N}$-body Liouville equation follows, in fact, as a straightforward consequence of the axioms of classical statistical mechanics [1].

Despite these premises, however, the case of a finite Boltzmann-Sinai CDS, which is characterized by a finite number of particles $N$ and/or a finite-size of the hardspheres and/or a dense or locally dense system, is more subtle and-as explained below-even unprecedented since it has actually remained unsolved to date. The reasons are as follows. First, Boltzmann and Grad kinetic theories are inapplicable to the finite Boltzmann-Sinai CDS. Second, the Boltzmann-Shannon entropy associated with an arbitrary particular solution $\rho^{(N)}(t) \equiv \rho^{(N)}\left(\mathbf{x}_{1}, t\right)$ of the Master kinetic equation, i.e., the functional $S\left(\rho_{1}^{(N)}(t)\right) \equiv M_{X_{E}}\left(\rho_{1}^{(N)}(t)\right)$, in contrast to $S\left(\rho_{1}(t)\right) \equiv M_{X_{E}}\left(\rho_{1}(t)\right)$, is exactly conserved in the sense that identically

$$
\frac{\partial S\left(\rho_{1}^{(N)}(t)\right)}{\partial t} \equiv 0
$$

must hold. As a consequence the validity itself of Boltzmann $\mathrm{H}$-theorem breaks down in the case of the Master kinetic equation. Third, an additional motivation is provided by the conjecture that both PMI and DKE might occur only if the Boltzmann-Grad limit is actually performed, i.e., only in validity of Boltzmann equation and $\mathrm{H}$-theorem.

Hence the question which arises is whether in the case of a finite Boltzmann-Sinai CDS the phenomenon of DKE may still arise. Strong indications seem to be hinting at such a possibility. In this regard the example-case which refers to the statistical description of a Navier-Stokes fluid described by the incompressible Navier-Stokes equations (INSE) in terms of the Master kinetic equation is relevant and suggests that this may be indeed the case. In fact, thanks also to comparisons with the mean-field inverse kinetic approach to INSE [16], in such a case the decay of the fluid velocity field occurring in a bounded domain necessarily demands the existence of DKE. In other words, in the limit $t \longrightarrow+\infty$ the 1-body PDF must decay uniformly to the stationary and spatially uniform Maxwellian PDF (6).

However, besides the construction of the kinetic equation appropriate for such a case, a further unsolved issue lies in the determination of the functional class $\left\{\rho_{1}^{(N)}\left(\mathbf{x}_{1}, t\right)\right\}$ for which both PMI and DKE should/might be realized. In particular, the possible occurrence of both PMI and DKE should correspond to suitably smooth, but nonetheless still arbitrary, initial conditions $\left\{\rho_{1}^{(N)}\left(\mathbf{x}_{1}, t_{o}\right)\right\}$. These should warrant that in the limit $t \longrightarrow+\infty, \rho_{1}^{(N)}\left(\mathbf{x}_{1}, t\right)$ uniformly converges to the spatially homogeneous and stationary Maxwellian PDF $\rho_{1 M}\left(\mathbf{v}_{1}\right)$ (6). Such a result, however, is highly nontrivial since it should rely on the establishment of a global existence theorem for the same 1 -body $\operatorname{PDF} \rho_{1}^{(N)}\left(\mathbf{x}_{1}, t\right)$-namely, holding in the whole time axis $I \equiv \mathbb{R}$, besides the same 1 -body phase space $\Gamma_{1}$-for the involved kinetic equation which is associated with the $S_{N}$-CDS. In the context of the Boltzmann equation in particular, despite almost-endless efforts this task has actually not been accomplished yet, the obstacle being intrinsically related to the asymptotic nature of the Boltzmann equation [13]. In fact for the same equation it is not known in satisfactory generality whether smooth enough solutions of the same equation exist which satisfy the $H$-theorem inequality and decay asymptotically to kinetic equilibrium $[23,30]$.

1.2. Goals and Organization of the Paper. Based on these premises, the crucial new results that we intend to display in this paper concern the proof-of-principle of two phenomena which are expected to characterize the statistical description of finite $N$-body hard-sphere systems and therefore should lay at the very foundation of classical statistical mechanics and kinetic theory alike. These are related to the physical conditions for the possible occurrence of both PMI and the consequent one represented by the possible occurrence of DKE which should characterize the kinetic PDF in these systems. These phenomena are well known to occur in the case of dilute hard-sphere systems, i.e., in the BoltzmannGrad limit. In particular, for an exhaustive treatment of the related issues which arise in the context of the ab initio theory we refer to discussions reported in [4]. Nevertheless, as indicated above, their existence in the case of finite hardsphere systems is partly motivated by a previous investigation dealing with the kinetic description incompressible NavierStokes granular fluids [33].

Therefore, main goal of the paper is to show that these properties actually emerge as necessary implications of the $\mathrm{ab}$ initio theory of CSM. Incidentally, in doing so, the Master kinetic equation must be necessarily adopted. In fact, the finiteness requirement on the $S_{N}$-CDS rules out for further possible consideration either the Boltzmann or the Enskog kinetic equations, these equations being inapplicable to the treatment of systems of this type [3]. Specifically, in the following the case $N>2$ is considered everywhere, which is by far the most physically relevant one. In this occurrence, in fact, nontrivial 2-body occupation coefficients arise (see related notations which are applicable for $N>2$ recalled in Appendices A and B below). For completeness the case $N=2$ is nevertheless briefly discussed in Appendix D.

For this purpose, first, in Section 2, the MKI functional is explicitly determined. We display in particular its construction method (see No.\#1- \#4 MKI Prescriptions). Based on the theory of the Master kinetic equation earlier developed [3] 
and suitable integral and differential identities (see Appendices $\mathrm{A}, \mathrm{B}$, and $\mathrm{C}$ ), the properties of the MKI functional are investigated. These concern in particular the establishment of appropriate inequalities holding for the same functional (Theorem 1, Section 2.1), the signature of the time derivative of the same functional (Theorem 2, Section 2.2) and the property of DKE holding for a suitable class of 1-body PDFs (Theorem 3, Section 2.3). In the subsequent Sections 3 and 4, the issue of the consistency of the phenomena of PMI and DKE with microscopic dynamics is posed together with the physical interpretation and implications of the theory. The goal is to investigate the relationship of the DKE theory developed here with the microscopic reversibility principle and the Poincaré recurrence theorem. Finally in Section 5 the conclusions of the paper are drawn and possible applications/developments of the theory are pointed out.

\section{Axiomatic Prescription of the MKI Functional}

In view of the considerations given above in this section the problem is posed of the explicit realization of the MKI functional in terms of suitable axiomatic prescriptions. The same functional, denoted $I_{M}\left(\rho_{1}^{(N)}(t)\right)$, should depend on the 1 -body $\operatorname{PDF} \rho_{1}^{(N)}(t)$, with $\rho_{1}^{(N)}(t) \equiv \rho_{1}^{(N)}\left(\mathbf{x}_{1}, t\right)$ being identified with a particular solution of the Master kinetic equation (see (A.4) in Appendix A holding for $N>2$ and Appendix D for the case $N=2$ ).

Unlike Boltzmann kinetic equation, the Master kinetic equation actually deals with the treatment of finite hardsphere $N$-body systems, i.e., in which both the number of particles $N$ and their diameter $\sigma$ remain finite [3]. To achieve such a goal suitably prescribed physical collision boundary conditions (CBC) of the $N$-body PDF need to be adopted. More precisely, this concerns the prescription for arbitrary collision events of the relationship between incoming (-) and outgoing (+) PDFs, i.e., respectively, the left and right limits $\rho^{( \pm)(N)}\left(\mathbf{x}^{( \pm)}\left(t_{i}\right), t_{i}\right)=\lim _{t \rightarrow t_{i}^{( \pm)}} \rho^{(N)}(\mathbf{x}(t), t)$, with $\mathbf{x}^{( \pm)}\left(t_{i}\right)=\lim _{t \rightarrow t_{i}^{( \pm)}} \mathbf{x}(t)$ denoting the corresponding incoming (-) and outgoing (+) states. In particular, upon invoking due to causality the assumption of left-continuity, i.e., the requirement

$$
\rho^{(-)(N)}\left(\mathbf{x}^{(-)}\left(t_{i}\right), t_{i}\right) \equiv \rho^{(N)}\left(\mathbf{x}^{(-)}\left(t_{i}\right), t_{i}\right),
$$

the incoming PDF is required to coincide with the same $N$-body PDF evaluated in terms of the incoming state and time $[1,3]$. Hence, as recalled in Appendix C (see also [2]) from (C.1) it follows that the so-called causal form of the modified collision boundary condition (MCBC [2])

$$
\rho^{(+)(N)}\left(\mathbf{x}^{(+)}\left(t_{i}\right), t_{i}\right)=\rho^{(N)}\left(\mathbf{x}^{(+)}\left(t_{i}\right), t_{i}\right)
$$

is mandatory. A further important requirement concerns precisely setting also the related functional class of admissible solutions $\left\{\rho_{1}^{(N)}\left(\mathbf{x}_{1}, t\right)\right\}$ in such a way that, besides $\rho_{1}^{(N)}(t)$, also the same functional $I_{M}\left(\rho_{1}^{(N)}(t)\right)$ exists globally for arbitrary $t \in I \equiv \mathbb{R}$. For definiteness, we shall consider for this purpose the case of 1-body PDFs which satisfy the initial condition

$$
\rho_{1}^{(N)}\left(t_{o}\right) \equiv \rho_{1}^{(N)}\left(\mathbf{x}_{1}, t_{o}\right)=\rho_{1(o)}^{(N)}\left(\mathbf{x}_{1}\right),
$$

with $\rho_{1(o)}^{(N)}\left(\mathbf{x}_{1}\right)$ belonging to the functional class of stochastic 1-body PDFs $\left\{\rho_{1}^{(N)}\left(t_{o}\right)\right\}$. For a generic $t \in I$ belonging to the time axis $I \equiv \mathbb{R}$ this is the ensemble of 1 -body PDFs $\rho_{1}^{(N)}(t)$ which are, respectively, (A) smoothly differentiable; (B) strictly positive; (C) summable, in the sense that the velocity-or phase-space-moments for the same PDF exist which correspond either to arbitrary monomial functions of $\mathbf{v}_{1}$ (or its components $v_{1 i}$, for $i=1,2,3$ ) or to the entropy density $\ln \rho_{1}^{(N)}(t)$, thus yielding the Boltzmann-Shannon (BS) entropy evaluated in terms of $\rho_{1}^{(N)}(t)$.

Concerning the choice of the setting $\left\{\rho_{1}^{(N)}\left(t_{o}\right)\right\}$ the following remarks are in order. As a first remark, the previous requirements $(\mathrm{A}),(\mathrm{B})$, and $(\mathrm{C})$ for $\left\{\rho_{1}^{(N)}\left(t_{o}\right)\right\}$, together with validity of MCBC (14), actually should warrant that the corresponding solution of the Master kinetic equation $\rho_{1}^{(N)}(t)$ exists globally in the extended phase-space $\left(\mathbf{x}_{1}, t\right) \in \Gamma_{1} \times I$ and that for all $t \in I$ the same PDF belongs to the class of stochastic PDFs $\left\{\rho_{1}^{(N)}\left(\mathbf{x}_{1}, t\right)\right\}$ indicated above and also fulfills identically the constant H-theorem (12). Indeed, one can show [13] that global existence of solutions for the Master kinetic equation follows in elementary way from the $N$-body Liouville equation. Indeed, an arbitrary 1-body PDF which is a particular solution of the Master kinetic equation realizes by construction also a particular factorized solution of the $N$-body Liouville equation, i.e., of the N-body PDF [3]. The same PDF evolves uniquely in time along arbitrary phasespace Lagrangian trajectories, its Lagrangian time evolution being determined at arbitrary collision times by MCBC (14) [13].

As a second remark, the validity of assumptions (A) and (B) for $\rho_{1(o)}^{(N)}\left(\mathbf{x}_{1}\right)$ and $\rho_{1}^{(N)}\left(\mathbf{x}_{1}, t\right)$ implies also suitable assumptions to apply all $t \in I$ for the local characteristic scale length $L_{\rho}$ which characterize the same $\operatorname{PDF} \rho_{1}^{(N)}\left(\mathbf{x}_{1}, t\right)$. More precisely, this is associated with the spatial variations of the 1 -body PDF prescribed as

$$
L_{\rho}(t)=\inf _{\mathbf{x}_{1} \in \Gamma_{1}}\left\{\left|\frac{\partial \ln \rho_{1}^{(N)}\left(\mathbf{x}_{1}, t\right)}{\partial \mathbf{r}_{1}}\right|^{-1}\right\},
$$

which necessarily assumed nonzero at all time $t \in I$. Hence $\partial \ln \rho_{1}^{(N)}\left(\mathbf{x}_{1}, t\right) / \partial \mathbf{r}_{1}$ is assumed to be bounded for all $\left(\mathbf{x}_{1}, t\right)$ spanning the extended 1 -body phase space $\Gamma_{1(1)} \times I$.

Finally, as a third remark (see also the further related discussion in Theorem 2 below), the previous requirements are expected to warrant also the global existence of the MKI functional $I_{M}\left(\rho_{1}^{(N)}(t)\right)$, so that $\left\{\rho_{1}^{(N)}\left(\mathbf{x}_{1}, t\right)\right\}$ effectively realizes the functional class of admissible solutions indicated above.

Given these premises let us pose now the problem of the identification of the functional $I_{M}\left(\rho_{1 o}^{(N)}\left(\mathbf{x}_{1}\right)\right)$, based on the introduction of 'ad hoc' physical requirements, to be referred to here as MKI Prescriptions No.\#1-\#4. The prescriptions are as follows: 
(i) MKI Prescription No.\#1: the first one is that the functional $I_{M}\left(\rho_{1}^{(N)}(t)\right)$ should be determined in such a way that the existence of $I_{M}\left(\rho_{1 o}^{(N)}\left(\mathbf{x}_{1}\right)\right)$ at a suitable initial time $t_{o} \in I$ should warrant also that $I_{M}\left(\rho_{1}^{(N)}(t)\right)$ must necessarily exist globally in the future, i.e., for all $t \geq t_{o}$. As a consequence the functional class $\left\{\rho_{1}^{(N)}\left(\mathbf{x}_{1}, t\right)\right\}$ must be suitably prescribed.

(ii) MKI Prescription No.\#2: second, we shall require that $I_{M}\left(\rho_{1}^{(N)}(t)\right)$ is real, nonnegative, and bounded in the sense that

$$
0 \leq I_{M}\left(\rho_{1}^{(N)}(t)\right) \leq 1
$$

This implies that $I_{M}\left(\rho_{1}^{(N)}(t)\right)$ can be interpreted as an information measure associated with the 1-body $\operatorname{PDF} \rho_{1}^{(N)}(t) \equiv \rho_{1}^{(N)}\left(\mathbf{x}_{1}, t\right)$. For this reason the previous inequalities will be referred to as information-measure inequalities.

(iii) MKI Prescription No.\#3: third, for consistency with the property of macroscopic irreversibility, $I_{M}\left(\rho_{1}^{(N)}(t)\right)$ is prescribed in terms of a smoothly timedifferentiable and monotonically time-decreasing functional in the sense that in the same time-subset the inequality

$$
\frac{\partial}{\partial t} I_{M}\left(\rho_{1}^{(N)}(t)\right) \leq 0
$$

should identically apply $\forall t \geq t_{o}$, so that

$$
0 \leq I_{M}\left(\rho_{1}^{(N)}(t)\right) \leq I_{M}\left(\rho_{1 o}^{(N)}\left(\mathbf{x}_{1}\right)\right) \leq 1 .
$$

This implies that $I_{M}\left(\rho_{1}^{(N)}(t)\right)$ is also globally defined for all $t \in I \equiv \mathbb{R}$ with $t \gtrsim t_{o}$. In addition, if $\left.(\partial / \partial t) I_{M}\left(\rho_{1}^{(N)}(t)\right)\right|_{t=t_{o}} \neq 0$, without loss of generality its initial value $I_{M}\left(\rho_{1 o}^{(N)}\left(\mathbf{x}_{1}\right)\right)$, can always be set such that

$$
I_{M}\left(\rho_{1 o}^{(N)}\left(\mathbf{x}_{1}\right)\right)=1
$$

(iv) MKI Prescription No.\#4: fourth, in order to warrant the existence of DKE we shall require the functional $I_{M}\left(\rho_{1}^{(N)}(t)\right)$ to be prescribed in such a way that an arbitrary time $t \in I$, with $t \gtrsim t_{o}$, the vanishing of both $I_{M}\left(\rho_{1}^{(N)}(t)\right)$ and its time derivative $(\partial / \partial t) I_{M}\left(\rho_{1}^{(N)}(t)\right)$ should occur if and only if the 1-body PDF solution of the Master kinetic equation coincides with kinetic equilibrium. As a consequence, for the functional $I_{M}\left(\rho_{1}^{(N)}(t)\right)$ the following propositions should be equivalent:

$$
\begin{aligned}
I_{M}\left(\rho_{1}^{(N)}(t)\right) & =0 \\
\frac{\partial}{\partial t} I_{M}\left(\rho_{1}^{(N)}(t)\right) & =0 \\
& \Uparrow \\
\rho_{1}^{(N)}\left(\mathbf{x}_{1}, t\right) & \equiv \rho_{1 M}^{(N)}\left(\mathbf{v}_{1}\right),
\end{aligned}
$$

with $\rho_{1 M}^{(N)}\left(\mathbf{v}_{1}\right)$ being a kinetic equilibrium PDF of the form (6).

The immediate obvious implication of the previous prescriptions is that-provided a nontrivial realization of the MKI can be found in the functional class $\left\{\rho_{1 o}^{(N)}\left(\mathbf{x}_{1}\right)\right\}$-the existence of both PMI and DKE for the Master kinetic equation is actually established. In the sequel the goal is to show, in particular, that the MKI functional can be identified by means of the prescription

$$
I_{M}\left(\rho_{1}^{(N)}(t), \mathbf{b}\right) \equiv \frac{K_{M}\left(\rho_{1}^{(N)}(t), \mathbf{b}\right)}{K_{M o}},
$$

where $K_{M}\left(\rho_{1}^{(N)}(t), \mathbf{b}\right)$ and $K_{M o}$ denote, respectively, a suitable (and possibly nonunique) moment-dependent phase-space functional and an appropriate normalization constant to be chosen in such a way to satisfy all the MKI prescriptions indicated above. In particular, as shown below, an admissible choice for $K_{M}\left(\rho_{1}^{(N)}(t), b\right)$ and $K_{M o}$ is provided by

$$
\begin{aligned}
& K_{M}\left(\rho_{1}^{(N)}(t), \mathbf{b}\right)=-\int_{\Gamma_{1(1)}} d \mathbf{x}_{1} \bar{\Theta}_{1}^{(\partial \Omega)}\left(\overline{\mathbf{r}}_{1}\right) M\left(\mathbf{v}_{1}, \mathbf{b}\right) \\
& \cdot \frac{\rho_{1}^{(N)}\left(\mathbf{x}_{1}, t\right)}{\widehat{\rho}_{1}^{(N)}\left(\mathbf{x}_{1}, t\right)} \frac{\partial^{2} \widehat{\rho}_{1}^{(N)}\left(\mathbf{x}_{1}, t\right)}{\partial \mathbf{r}_{1} \cdot \partial \mathbf{r}_{1}}, \\
& K_{M o}=\sup \left\{1, K_{M}\left(\rho_{1 o}^{(N)}\left(\mathbf{x}_{1}\right), \mathbf{b}\right)\right\},
\end{aligned}
$$

while $M\left(\mathbf{v}_{1}, \mathbf{b}\right)$ denotes the directional kinetic energy (along the unit vector $\mathbf{b}$ ) carried by particle 1 , namely, the dynamical variable

$$
M\left(\mathbf{v}_{1}, \mathbf{b}\right) \equiv\left(\mathbf{v}_{1} \cdot \mathbf{b}\right)^{2},
$$

with $\mathbf{b}$ denoting a still arbitrary constant unit vector. Hence,

$$
M\left(\mathbf{v}_{1}, \mathbf{v}_{2}, \mathbf{b}\right)=\frac{1}{2}\left[M\left(\mathbf{v}_{1}, \mathbf{b}\right)+M\left(\mathbf{v}_{2}, \mathbf{b}\right)\right]
$$

identifies the corresponding total directional kinetic energy carried by particles 1 and 2 . Here the remaining notation is standard. Thus, $\rho_{1}^{(N)}(t) \equiv \rho_{1}^{(N)}\left(\mathbf{x}_{1}, t\right), \rho_{1 o}^{(N)}\left(\mathbf{x}_{1}\right)$, and $\widehat{\rho}_{1}^{(N)}(t) \equiv$ $\hat{\rho}_{1}^{(N)}\left(\mathbf{x}_{1}, t\right)$ are, respectively, the 1 -body PDF solution of the initial problem associated with the Master kinetic equation (see (A.4) in Appendix A), the initial PDF, and the renormalized 1-body PDF

$$
\hat{\rho}_{1}^{(N)}\left(\mathbf{x}_{1}, t\right) \equiv \frac{\rho_{1}^{(N)}\left(\mathbf{x}_{1}, t\right)}{k_{1}^{(N)}\left(\mathbf{r}_{1}, t\right)},
$$

while furthermore $k_{1}^{(N)}\left(\mathbf{r}_{1}, t\right)$ is the 1-body occupation coefficient recalled in Appendix B (see (B.1)). As a consequence in the previous equation it follows that $\rho_{1}^{(N)}\left(\mathbf{x}_{1}, t\right) / \widehat{\rho}_{1}^{(N)}\left(\mathbf{x}_{1}, t\right) \equiv$ $k_{1}^{(N)}\left(\mathbf{r}_{1}, t\right)$. Furthermore, $\bar{\Theta}_{1}^{(\partial \Omega)}\left(\overline{\mathbf{r}}_{1}\right)$ is the boundary theta function given by (A.10) (see Appendix A). Finally, regarding the initial value $K_{M}\left(\rho_{1 o}^{(N)}\left(\mathbf{x}_{1}\right), \mathbf{b}\right)$ it follows that if, respectively, $K_{M}\left(\rho_{1 o}^{(N)}\left(\mathbf{x}_{1}\right), \mathbf{b}\right) \geq 1$ or

$$
0 \leq K_{M}\left(\rho_{1 o}^{(N)}\left(\mathbf{x}_{1}\right), \mathbf{b}\right)<1,
$$


then correspondingly one obtains, consistent with (19), that the initial value of MKI functional $I_{M}\left(\rho_{1 o}^{(N)}, \mathbf{b}\right)$ is

$$
I_{M}\left(\rho_{1 o}^{(N)}, \mathbf{b}\right)=\left\{\begin{array}{l}
1, \\
K_{M}\left(\rho_{1 o}^{(N)}\left(\mathbf{x}_{1}\right), \mathbf{b}\right) .
\end{array}\right.
$$

2.1. Proof of the Nonnegativity of the MKI Information Measure. The strategy adopted for the proof of the MKI Prescriptions No.\#1 and No.\#2 is to show initially the validity of the information-measure left inequality in (17), namely, that $I_{M}\left(\rho_{1}^{(N)}(t), \mathbf{b}\right)$ cannot acquire negative values for arbitrary $t \geq t_{o}$. The result is established by the following theorem.

Theorem 1 (nonnegativity of $K_{M}\left(\rho_{1 o}^{(N)}\left(\mathbf{x}_{1}\right), \mathbf{b}\right), K_{M}\left(\rho_{1}^{(N)}(t), \mathbf{b}\right)$, and $\left.I_{M}\left(\rho_{1}^{(N)}(t), \mathbf{b}\right)\right)$. Let us assume that $\rho_{1}^{(N)}\left(\mathbf{x}_{1}, t\right)$ is an arbitrary stochastic and suitably smoothly differentiable, particular solution of the Master kinetic equation (A.4) prescribed so that the integral (23) expressed in terms of the initial PDF, namely, $K_{M}\left(\rho_{1 o}^{(N)}\left(\mathbf{x}_{1}\right), \mathbf{b}\right)$, is nonvanishing. Then, it follows necessarily that

(i) Proposition $P 1_{1}$ :

$$
K_{M}\left(\rho_{1 o}^{(N)}\left(\mathbf{x}_{1}\right), \mathbf{b}\right)>0 .
$$

(ii) Proposition $\mathrm{Pl}_{2}$ : the corresponding time-evolved functional $K_{M}\left(\rho_{1}^{(N)}(t), \mathbf{b}\right)$ for all $t \in I$ with $t>t_{o}$ is such that

$$
K_{M}\left(\rho_{1}^{(N)}(t), \mathbf{b}\right) \geq 0 .
$$

(iii) Proposition $\mathrm{Pl}_{3}$ : for all $t \in I$ with $t>t_{0}$ the functional $I_{M}\left(\rho_{1}^{(N)}(t), \mathbf{b}\right)$ fulfills the inequality

$$
I_{M}\left(\rho_{1}^{(N)}(t), \mathbf{b}\right) \geq 0 .
$$

(iv) Proposition $\mathrm{P1}_{4}$ : the following necessary and sufficient condition holds at a given time $t \in I$ with $t \geq t_{o}$ :

$$
\begin{aligned}
K_{M}\left(\rho_{1}^{(N)}(t), \mathbf{b}\right) & =0 \Longleftrightarrow \\
\rho_{1}^{(N)}\left(\mathbf{x}_{1}, t\right) & \equiv \rho_{1 M}^{(N)}\left(\mathbf{v}_{1}\right) .
\end{aligned}
$$

Proof. One first notices that $K_{M}\left(\rho_{1}^{(N)}(t), \mathbf{b}\right)$ can be equivalently written in the form

$$
\begin{aligned}
& K_{M}\left(\rho_{1}^{(N)}(t), \mathbf{b}\right) \equiv-\int_{\Gamma_{1(1)}} d \mathbf{x}_{1} \bar{\Theta}_{1}^{(\partial \Omega)}\left(\overline{\mathbf{r}}_{1}\right) M\left(\mathbf{v}_{1}, \mathbf{b}\right) \\
& \cdot k_{1}^{(N)}\left(\mathbf{r}_{1}, t\right) \frac{\partial^{2} \widehat{\rho}_{1}^{(N)}\left(\mathbf{x}_{1}, t\right)}{\partial \mathbf{r}_{1} \cdot \partial \mathbf{r}_{1}}
\end{aligned}
$$

where in order that the same functional exists it is obvious that the renormalized 1-body $\operatorname{PDF} \hat{\rho}_{1}^{(N)}\left(\mathbf{x}_{1}, t\right)$ must be of class $C^{(2)}$. Integrating by parts and noting that the gradient term $\partial \bar{\Theta}_{1}^{(\partial \Omega)}(\overline{\mathbf{r}}) / \partial \mathbf{r}_{1}$ gives a vanishing contribution to the phasespace integral, this yields equivalently

$$
\begin{aligned}
K_{M} & \left(\rho_{1}^{(N)}(t), \mathbf{b}\right) \\
\equiv & \int_{\Gamma_{1(1)}} d \mathbf{x}_{1} \bar{\Theta}_{1}^{(\partial \Omega)}\left(\overline{\mathbf{r}}_{1}\right) M\left(\mathbf{v}_{1}, \mathbf{b}\right) \frac{\partial k_{1}^{(N)}\left(\mathbf{r}_{1}, t\right)}{\partial \mathbf{r}_{1}} \\
& \cdot \frac{\partial \widehat{\rho}_{1}^{(N)}\left(\mathbf{x}_{1}, t\right)}{\partial \mathbf{r}_{1}} .
\end{aligned}
$$

Therefore, upon invoking (B.9) reported in Appendix B, direct substitution delivers

$$
\begin{aligned}
& K_{M}\left(\rho_{1}^{(N)}(t), \mathbf{b}\right)=(N-1) \int_{\Gamma_{1(1)}} d \mathbf{x}_{1} \bar{\Theta}_{1}^{(\partial \Omega)}\left(\overline{\mathbf{r}}_{1}\right) \\
& \cdot M\left(\mathbf{v}_{1}, \mathbf{b}\right) \frac{\partial \hat{\rho}_{1}^{(N)}\left(\mathbf{x}_{1}, t\right)}{\partial \mathbf{r}_{1}} \cdot \int_{\Gamma_{1(2)}} d \mathbf{x}_{2} \mathbf{n}_{12} \\
& \quad \times \delta\left(\left|\mathbf{r}_{2}-\mathbf{r}_{1}\right|-\sigma\right) \bar{\Theta}_{2}^{(\partial \Omega)}\left(\overline{\mathbf{r}}_{2}\right) \widehat{\rho}_{1}^{(N)}\left(\mathbf{x}_{2}, t\right) \\
& \cdot k_{2}^{(N)}\left(\mathbf{r}_{1}, \mathbf{r}_{2}, t\right) .
\end{aligned}
$$

Next, invoking the identity $\mathbf{n}_{12} \delta\left(\left|\mathbf{r}_{2}-\mathbf{r}_{1}\right|-\sigma\right)=-\left(\partial / \partial \mathbf{r}_{2}\right) \bar{\Theta}\left(\mid \mathbf{r}_{2}\right.$ $\left.-\mathbf{r}_{1} \mid-\sigma\right)$ and noting again that $\left(\partial / \partial \mathbf{r}_{2}\right) \bar{\Theta}_{2}^{(\partial \Omega)}(\overline{\mathbf{r}})$ gives vanishing contribution, one can perform a further integration by parts with respect to $\mathbf{r}_{2}$. This permits to cast the rhs of previous equation in the form

$$
\begin{aligned}
K_{M}\left(\rho_{1}^{(N)}(t), \mathbf{b}\right) \equiv & K_{M}^{(1)}\left(\rho_{1}^{(N)}\left(\mathbf{x}_{1}, t\right), \mathbf{b}\right) \\
& +\Delta K_{M}^{(1)}\left(\rho_{1}^{(N)}\left(\mathbf{x}_{1}, t\right), \mathbf{b}\right) .
\end{aligned}
$$

Here the two terms on the rhs of (36) are defined as follows: (1) the first term $K_{M}^{(1)}\left(\rho_{1}^{(N)}(t), \mathbf{b}\right)$ is symmetric and nonnegative, so that it can be expressed so to carry the total directional kinetic energy $M\left(\mathbf{v}_{1}, \mathbf{v}_{2}, \mathbf{b}\right)$ of particles 1 and 2 (see (25)). Hence, it takes the form

$$
\begin{aligned}
& K_{M}^{(1)}\left(\rho_{1}^{(N)}\left(\mathbf{x}_{1}, t\right), \mathbf{b}\right)=(N-1) \int_{\Gamma_{1(1)}} d \mathbf{x}_{1} \\
& \cdot \int_{\Gamma_{1(2)}} d \mathbf{x}_{2} \bar{\Theta}_{1}^{(\partial \Omega)}\left(\overline{\mathbf{r}}_{1}\right) \bar{\Theta}_{2}^{(\partial \Omega)}\left(\overline{\mathbf{r}}_{2}\right) \times \frac{\partial \widehat{\rho}_{1}^{(N)}\left(\mathbf{x}_{1}, t\right)}{\partial \mathbf{r}_{1}} \\
& \quad \cdot \frac{\partial \widehat{\rho}_{1}^{(N)}\left(\mathbf{x}_{2}, t\right)}{\partial \mathbf{r}_{2}} k_{2}^{(N)}\left(\mathbf{r}_{1}, \mathbf{r}_{2}, t\right) M\left(\mathbf{v}_{1}, \mathbf{v}_{2}, \mathbf{b}\right) \\
& \cdot \bar{\Theta}\left(\left|\mathbf{r}_{2}-\mathbf{r}_{1}\right|-\sigma\right) .
\end{aligned}
$$

(2) The second term $\Delta K_{M}^{(1)}\left(\rho_{1}^{(N)}\left(\mathbf{x}_{1}, t\right), \mathbf{b}\right)$ reads instead

$$
\begin{aligned}
& \Delta K_{M}^{(1)}\left(\rho_{1}^{(N)}\left(\mathbf{x}_{1}, t\right), \mathbf{b}\right) \equiv(N-1) \\
& \quad \cdot \int_{\Gamma_{1(1)}} d \mathbf{x}_{1} \bar{\Theta}_{1}^{(\partial \Omega)}\left(\overline{\mathbf{r}}_{1}\right) \frac{\partial \widehat{\rho}_{1}^{(N)}\left(\mathbf{x}_{1}, t\right)}{\partial \mathbf{r}_{1}} \cdot \int_{\Gamma_{1(2)}} d \mathbf{x}_{2}
\end{aligned}
$$




$$
\begin{aligned}
& \times \bar{\Theta}_{2}^{(\partial \Omega)}\left(\overline{\mathbf{r}}_{2}\right) M\left(\mathbf{v}_{1}, \mathbf{b}\right) \bar{\Theta}\left(\left|\mathbf{r}_{2}-\mathbf{r}_{1}\right|-\sigma\right) \hat{\rho}_{1}^{(N)}\left(\mathbf{x}_{2},\right. \\
& \text { t) } \frac{\partial}{\partial \mathbf{r}_{2}} k_{2}^{(N)}\left(\mathbf{r}_{1}, \mathbf{r}_{2}, t\right),
\end{aligned}
$$

where $\left(\partial / \partial \mathbf{r}_{2}\right) k_{2}^{(N)}\left(\mathbf{r}_{1}, \mathbf{r}_{2}, t\right)$ is given by the differential identity (B.10) reported in Appendix B. Thus, upon invoking the identity $\mathbf{n}_{23} \delta\left(\left|\mathbf{r}_{3}-\mathbf{r}_{2}\right|-\sigma\right)=-\left(\partial / \partial \mathbf{r}_{3}\right) \bar{\Theta}\left(\left|\mathbf{r}_{3}-\mathbf{r}_{2}\right|-\right.$ $\sigma)$, one notices that an integration by parts can be performed also with respect to $\mathbf{r}_{3}$. This means that a procedure analogous to the one used for the calculation of $\partial k_{1}^{(N)}\left(\mathbf{r}_{1}, t\right) / \partial \mathbf{r}_{1}$ can be invoked and iterated at all orders, i.e., up to the $(N-1)$-body occupation coefficient (see (B.11) in Appendix B). As a consequence the functional $K_{M}\left(\rho_{1}^{(N)}(t), \mathbf{b}\right)$ can be represented in terms of a finite sum of the form $K_{M}\left(\rho_{1}^{(N)}(t), \mathbf{b}\right) \equiv \sum_{j=1, N-1} K_{M}^{(j)}\left(\rho_{1}^{(N)}\left(\mathbf{x}_{1}, t\right), \mathbf{b}\right)$ in which each term of the sum $K_{M}^{(j)}\left(\rho_{1}^{(N)}\left(\mathbf{x}_{1}, t\right), \mathbf{b}\right)$ is nonnegative and symmetric. This implies therefore that the same functional $K_{M}\left(\rho_{1}^{(N)}(t), \mathbf{b}\right)$ can be cast in the form

$$
\begin{aligned}
& K_{M}\left(\rho_{1}^{(N)}(t), \mathbf{b}\right)=\int_{\Gamma_{1(1)}} d \mathbf{x}_{1} \\
& \cdot \int_{\Gamma_{1(2)}} d \mathbf{x}_{2} \bar{\Theta}_{1}^{(\partial \Omega)}\left(\overline{\mathbf{r}}_{1}\right) \bar{\Theta}_{2}^{(\partial \Omega)}\left(\overline{\mathbf{r}}_{2}\right) M\left(\mathbf{v}_{1}, \mathbf{v}_{2}, \mathbf{b}\right) \\
& \quad \times F\left(\mathbf{r}_{1}, \mathbf{r}_{2}, t\right) \frac{\partial \widehat{\rho}_{1}^{(N)}\left(\mathbf{x}_{1}, t\right)}{\partial \mathbf{r}_{1}} \\
& \quad \cdot \frac{\partial \widehat{\rho}_{1}^{(N)}\left(\mathbf{x}_{2}, t\right)}{\partial \mathbf{r}_{2}} \bar{\Theta}\left(\left|\mathbf{r}_{2}-\mathbf{r}_{1}\right|-\sigma\right),
\end{aligned}
$$

with $M\left(\mathbf{v}_{1}, \mathbf{v}_{2}, \mathbf{b}\right) \geq 0$ being the total directional kinetic energy (25) and $F\left(\mathbf{r}_{1}, \mathbf{r}_{2}, t\right)$ a suitable real scalar kernel which is symmetric in the variables $\mathbf{r}_{1}$ and $\mathbf{r}_{2}$. Hence $K_{M}\left(\rho_{1}^{(N)}(t), \mathbf{b}\right)$ actually defines a nonnegative functional. This proves the validity of the inequality (31) (Proposition $P 1_{1}$ ).

In a similar way also the remaining propositions can be established. In fact, invoking (28) it follows that the inequalities (30) and (31)-and hence also Propositions $P 1_{2}$ and $\mathrm{Pl}_{3}-$ manifestly hold too. Finally, regarding the proof of Proposition $P 1_{4}$, one notices that $K_{M}\left(\rho_{1}^{(N)}(t), \mathbf{b}\right) \equiv 0$ if and only if identically $\left(\partial / \partial \mathbf{r}_{1}\right) \hat{\rho}_{1}^{(N)}\left(\mathbf{x}_{1}, t\right) \equiv 0$. Since $\hat{\rho}_{1}^{(N)}\left(\mathbf{x}_{1}, t\right)$ is by construction a solution of the Master kinetic equation it follows that this requires necessarily that $\hat{\rho}_{1}^{(N)}\left(\mathbf{x}_{1}, t\right)$ must coincide with the local Maxwellian $\rho_{1 M}^{(N)}\left(\mathbf{v}_{1}\right)$ (see (6)) and hence (32) must hold too under the same realization (Proposition $\left.\mathrm{P}_{4}\right)$.

The conclusion is therefore that the definition of the MKI functional (22) given above in terms of $K_{M}\left(\rho_{1}^{(N)}(t), \mathbf{b}\right)$ and $K_{M o}$ (see (23)) is indeed consistent with the physical prerequisites represented by the MKI Prescriptions No.\#1 and No.\#2.

2.2. Proof of PMI for the Master Kinetic Equation. The next step is to prove that the functional $I_{M}\left(\rho_{1 o}^{(N)}, \mathbf{b}\right)$ defined above (see (28)) indeed exhibits a monotonic time-decreasing behavior which is consistent with the MKI Prescriptions No.\#3 and No.\#4, which are realized respectively by

(i) the time derivative inequality (18) and the conditions of existence of kinetic equilibrium (21);

(ii) the validity of the inequality $I_{M}\left(\rho_{1}^{(N)}(t)\right) \leq 1$.

In order to reach the proofs of these properties let us preliminarily determine the variation across a binary collision occurring between particles 1 and 2 of the total directional kinetic energy $M\left(\mathbf{v}_{1}, \mathbf{v}_{2}, \mathbf{b}\right)$ (see (25)), namely, the phase-space scalar function $\Delta M\left(\mathbf{v}_{1}, \mathbf{v}_{2}, \mathbf{b}\right) \equiv M\left(\mathbf{v}_{1}^{(+)}, \mathbf{v}_{2}^{(+)}, \mathbf{b}\right)-$ $M\left(\mathbf{v}_{1} \mathbf{v}_{2}, \mathbf{b}\right)$. One obtains

$$
\begin{aligned}
\Delta M\left(\mathbf{v}_{1}, \mathbf{v}_{2}, \mathbf{b}\right)= & \mathbf{b} \cdot \mathbf{n}_{12}\left|\mathbf{n}_{12} \cdot \mathbf{v}_{12}^{(+)}\right| \mathbf{v}_{12}^{(+)} \cdot \mathbf{b} \\
& -\left(\mathbf{b} \cdot \mathbf{n}_{12}\right)^{2}\left(\mathbf{n}_{12} \cdot \mathbf{v}_{12}^{(+)}\right)^{2},
\end{aligned}
$$

the rhs being expressed in terms of the outgoing particle velocities $\left(\mathbf{v}_{1}^{(+)}, \mathbf{v}_{2}^{(+)}\right)$only. Then, the following proposition holds.

Theorem 2 (property of macroscopic irreversibility (master equation PMI theorem)). Let us assume that $\rho_{1}^{(N)}\left(\mathbf{x}_{1}, t\right) \equiv$ $\rho_{1}^{(N)}\left(\mathbf{r}_{1}, \mathbf{v}_{1}, t\right)$ is an arbitrary stochastic particular solution of the Master kinetic equation (A.4) with initial condition $\rho_{1 o}^{(N)}\left(\mathbf{x}_{1}\right)$ such that the integral $K_{M}\left(\rho_{1 o}^{(N)}\left(\mathbf{x}_{1}\right), \mathbf{b}\right)$ exists and is nonvanishing. Then it follows that

(i) Proposition P2 1 : one finds that for all $t \geq t_{o}$

$$
\begin{aligned}
& \frac{\partial}{\partial t} K_{M}\left(\rho_{1}^{(N)}(t), \mathbf{b}\right)=-(N-1) \sigma^{2} \int_{U_{1(1)}} d \mathbf{v}_{1} \int_{U_{1(2)}} d \mathbf{v}_{2} \\
& \cdot \int_{\Omega} d \mathbf{r}_{1} \bar{\Theta}_{1}^{(\partial \Omega)}\left(\overline{\mathbf{r}}_{1}\right) \int^{(-)} d \mathbf{s}_{21}\left|\mathbf{v}_{12}^{(+)} \cdot \mathbf{n}_{12}\right|\left(\mathbf{b} \cdot \mathbf{n}_{12}\right)^{2} \\
& \cdot\left(\mathbf{n}_{12} \cdot \mathbf{v}_{12}^{(+)}\right)^{2} \frac{\partial \hat{\rho}_{1}^{(N)}\left(\mathbf{r}_{1}, \mathbf{v}_{1}^{(+)}, t\right)}{\partial \mathbf{r}_{1}} \cdot \frac{\partial}{\partial \mathbf{r}_{2}} \\
& \cdot \widehat{\rho}_{1}^{(N)}\left(\mathbf{r}_{2}=\mathbf{r}_{1}+\sigma \mathbf{n}_{21}, \mathbf{v}_{2}^{(+)} t\right) \\
& \cdot k_{2}^{(N)}\left(\mathbf{r}_{1}, \mathbf{r}_{2}=\mathbf{r}_{1}+\sigma \mathbf{n}_{21}, t\right) \leq 0 .
\end{aligned}
$$

(ii) Proposition $\mathrm{P} 2_{2}$ : the inequality

$$
\frac{\partial}{\partial t} I_{M}\left(\rho_{1}^{(N)}(t), \mathbf{b}\right) \leq 0
$$

holds globally (i.e., identically for all $t \geq t_{o}$ ) so that necessarily $I_{M}\left(\rho_{1}^{(N)}(t), \mathbf{b}\right)$ is globally defined too, being also prescribed so that

$$
I_{M}\left(\rho_{1}^{(N)}(t), \mathbf{b}\right) \leq 1 .
$$

(iii) Proposition $P 2_{3}$ : one finds that a given time $t \in I$ with $t \geq t_{o}$

$$
\begin{aligned}
\frac{\partial}{\partial t} K_{M}\left(\rho_{1}^{(N)}(t), \mathbf{b}\right) & =0 \Longleftrightarrow \\
\rho_{1}^{(N)}\left(\mathbf{x}_{1}, t\right) & \equiv \rho_{1 M}^{(N)}\left(\mathbf{v}_{1}\right) .
\end{aligned}
$$


Proof. Consider first the proof of Proposition $P 2_{1}$ which requires evaluation of the partial time derivative $(\partial /$ $\partial t) K_{M}\left(\rho_{1}^{(N)}(t), \mathbf{b}\right)$. Upon invoking the first form of the Master kinetic equation (see (A.1) in Appendix A), explicit differentiation of $K_{M}\left(\rho_{1}^{(N)}(t)\right.$, b) delivers

$$
\begin{gathered}
\frac{\partial}{\partial t} K_{M}\left(\rho_{1}^{(N)}(t), \mathbf{b}\right)=-\int_{\Gamma_{1(1)}} d \mathbf{x}_{1} M\left(\mathbf{v}_{1}, \mathbf{b}\right) \bar{\Theta}_{1}^{(\partial \Omega)}\left(\overline{\mathbf{r}}_{1}\right) \\
\cdot k_{1}^{(N)}\left(\mathbf{r}_{1}, t\right)\left(-\mathbf{v}_{1} \cdot \frac{\partial}{\partial \mathbf{r}_{1}}\right) \frac{\partial^{2} \hat{\rho}_{1}^{(N)}\left(\mathbf{x}_{1}, t\right)}{\partial \mathbf{r}_{1} \cdot \partial \mathbf{r}_{1}}
\end{gathered}
$$$$
\frac{\partial}{\partial t} K_{M}-\left(f_{1_{(1)}^{(N)}}^{(N)} d \mathbf{x}_{1}, \mathbf{b}\right)\left(\mathbf{v}_{1}, \mathbf{b}\right) \bar{\Theta}_{1}^{(\partial \Omega)} \frac{\partial^{2} \widehat{\rho}_{1}^{(N)}\left(\mathbf{x}_{1}, t\right)}{\partial \mathbf{r}_{1} \cdot \partial \mathbf{r}_{1}}\left(\frac{\partial}{\partial t}\right)
$$$$
=-(N-1) \int_{\Gamma_{1(1)}} d \mathbf{x}_{1} M\left(\mathbf{v}_{1}, \mathbf{b}\right) \bar{\Theta}_{1}^{(\partial \Omega)}\left(\overline{\mathbf{r}}_{1}\right) \frac{\partial^{2} \widehat{\rho}_{1}^{(N)}\left(\mathbf{x}_{1}, t\right)}{\partial \mathbf{r}_{1} \cdot \partial \mathbf{r}_{1}} \int_{\bar{\Gamma}_{1(2)}} d \mathbf{x}_{2} \mathbf{v}_{12} \cdot \mathbf{n}_{12} \times \delta\left(\left|\mathbf{r}_{1}-\mathbf{r}_{2}\right|-\sigma\right) k_{2}^{(N)}\left(\mathbf{r}_{1}, \mathbf{r}_{2}, t\right) \widehat{\rho}_{1}^{(N)}\left(\mathbf{x}_{2}, t\right) .
$$

$$
\cdot k_{1}^{(N)}\left(\mathbf{r}_{1}, t\right)
$$

namely, upon integration by parts in the first integral on the rhs,

$$
\begin{aligned}
\frac{\partial}{\partial t} K_{M}\left(\rho_{1}^{(N)}(t), \mathbf{b}\right)=-\int_{\Gamma_{1(1)}} d \mathbf{x}_{1} M\left(\mathbf{v}_{1}, \mathbf{b}\right) \\
\quad \cdot \bar{\Theta}_{1}^{(\partial \Omega)} \frac{\partial^{2} \widehat{\rho}_{1}^{(N)}\left(\mathbf{x}_{1}, t\right)}{\partial \mathbf{r}_{1} \cdot \partial \mathbf{r}_{1}}\left(\frac{\partial}{\partial t}+\mathbf{v}_{1} \cdot \frac{\partial}{\partial \mathbf{r}_{1}}\right) k_{1}^{(N)}\left(\mathbf{r}_{1}, t\right) .
\end{aligned}
$$

Hence, thanks to the differential identity (B.12) it follows
Performing an integration by parts with respect to $\mathbf{r}_{1}$ and upon invoking the first differential identity (B.14) reported in Appendix B one obtains therefore

$$
\begin{aligned}
& \frac{\partial}{\partial t} K_{M}\left(\rho_{1}^{(N)}(t), \mathbf{b}\right)=W_{M}\left(\rho_{1}^{(N)}(t), \mathbf{b}\right) \\
& W_{M}\left(\rho_{1}^{(N)}(t), \mathbf{b}\right) \equiv \int_{\Gamma_{1(1)}} d \mathbf{x}_{1} M\left(\mathbf{v}_{1}, \mathbf{b}\right) \\
& \quad \cdot \bar{\Theta}_{1}^{(\partial \Omega)}\left(\overline{\mathbf{r}}_{1}\right) \frac{\partial \widehat{\rho}_{1}^{(N)}\left(\mathbf{x}_{1}, t\right)}{\partial \mathbf{r}_{1}}(N-1) \\
& \quad \times \int_{\bar{\Gamma}_{1(2)}} d \mathbf{x}_{2} \mathbf{v}_{12} \cdot \mathbf{n}_{12} \frac{\partial}{\partial \mathbf{r}_{1}}\left[\delta\left(\left|\mathbf{r}_{1}-\mathbf{r}_{2}\right|-\sigma\right)\right] \\
& \cdot k_{2}^{(N)}\left(\mathbf{r}_{1}, \mathbf{r}_{2}, t\right) \hat{\rho}_{1}^{(N)}\left(\mathbf{x}_{2}, t\right),
\end{aligned}
$$

where $\left(\partial / \partial \mathbf{r}_{1}\right)\left[\delta\left(\left|\mathbf{r}_{1}-\mathbf{r}_{2}\right|-\sigma\right)\right]=-\left(\partial / \partial \mathbf{r}_{2}\right)\left[\delta\left(\left|\mathbf{r}_{1}-\mathbf{r}_{2}\right|-\sigma\right)\right]$. Hence performing a further integration by parts with respect to $\mathbf{r}_{2}$ and using the second differential identity on (B.14) (see Appendix B) the previous equation finally yields

$$
\begin{aligned}
W_{M}\left(\rho_{1}^{(N)}(t), \mathbf{b}\right)= & (N-1) \int_{\Gamma_{1(1)}} d \mathbf{x}_{1} \bar{\Theta}_{1}^{(\partial \Omega)}\left(\overline{\mathbf{r}}_{1}\right) \\
& \cdot \int_{\bar{\Gamma}_{1(2)}} d \mathbf{x}_{2} \mathbf{v}_{12} \cdot \mathbf{n}_{12} M\left(\mathbf{v}_{1}, \mathbf{v}_{2}, \mathbf{b}\right) \\
& \times \delta\left(\left|\mathbf{r}_{1}-\mathbf{r}_{2}\right|-\sigma\right) \\
& \cdot k_{2}^{(N)}\left(\mathbf{r}_{1}, \mathbf{r}_{2}, t\right) \frac{\partial \widehat{\rho}_{1}^{(N)}\left(\mathbf{x}_{1}, t\right)}{\partial \mathbf{r}_{1}} \\
& \cdot \frac{\partial \widehat{\rho}_{1}^{(N)}\left(\mathbf{x}_{2}, t\right)}{\partial \mathbf{r}_{2}},
\end{aligned}
$$

where the symmetry property with respect to the exchange of states $\left(\mathbf{x}_{1}, \mathbf{x}_{2}\right)$ has been invoked. In the previous equation the integration on the Dirac delta can be performed at once letting

$$
\begin{aligned}
& \int_{\Gamma_{1(1)}} d \mathbf{x}_{1} \bar{\Theta}_{1}^{(\partial \Omega)} \int_{\Gamma_{1(2)}} d \mathbf{x}_{2} \delta\left(\left|\mathbf{r}_{1}-\mathbf{r}_{2}\right|-\sigma\right)=\sigma^{2} \int_{U_{1(1)}} d \mathbf{v}_{1} \\
& \cdot \int_{U_{1(2)}} d \mathbf{v}_{2} \int_{\Omega} d \mathbf{r}_{1} \bar{\Theta}_{1}^{(\partial \Omega)}\left(\overline{\mathbf{r}}_{1}\right) \\
& \cdot\left[\int^{(+)} d \mathbf{\Sigma}_{21}\left|\mathbf{v}_{12} \cdot \mathbf{n}_{12}\right|-\int^{(-)} d \mathbf{\Sigma}_{21}\left|\mathbf{v}_{12} \cdot \mathbf{n}_{12}\right|\right],
\end{aligned}
$$

where the solid-angle integrations in the two integrals on the rhs are performed, respectively, on the outgoing $(+)$ and incoming $(-)$ particles. Furthermore, it is obvious that thanks to the causal form of MCBC (see (C.3) in Appendix C) the integral on outgoing particles $\int^{(+)} d \Sigma_{21}$ can be transformed to a corresponding integration on incoming ones, namely, $\int^{(-)} d \Sigma_{21}$. Thus, the contributions in the two phase-space integrals only differ because of the variation $\Delta M\left(\mathbf{v}_{1}, \mathbf{v}_{2}, \mathbf{b}\right)$ of the total directional kinetic energy of particles 1 and 2 . This implies that

$$
\begin{gathered}
W_{M}\left(\rho_{1}^{(N)}(t), \mathbf{b}\right)=(N-1) \sigma^{2} \int_{U_{1(1)}} d \mathbf{v}_{1} \int_{U_{1(2)}} d \mathbf{v}_{2} \\
\cdot \int_{\Omega} d \mathbf{r}_{1} \bar{\Theta}_{1}^{(\partial \Omega)}\left(\overline{\mathbf{r}}_{1}\right) \int^{(-)} d \mathbf{\Sigma}_{21} \times\left|\mathbf{v}_{12} \cdot \mathbf{n}_{12}\right| \\
\cdot \Delta M\left(\mathbf{v}_{1}, \mathbf{v}_{2}, \mathbf{b}\right) \frac{\partial \widehat{\rho}_{1}^{(N)}\left(\mathbf{r}_{1}, \mathbf{v}_{1}^{(+)}, t\right)}{\partial \mathbf{r}_{1}}
\end{gathered}
$$




$$
\begin{aligned}
& \cdot \frac{\partial \widehat{\rho}_{1}^{(N)}\left(\mathbf{r}_{2}=\mathbf{r}_{1}+\sigma \mathbf{n}_{21}, \mathbf{v}_{2}^{(+)}, t\right)}{\partial \mathbf{r}_{2}} \\
& \times k_{2}^{(N)}\left(\mathbf{r}_{1}, \mathbf{r}_{2}=\mathbf{r}_{1}+\sigma \mathbf{n}_{21}, t\right),
\end{aligned}
$$

where the solid-angle integration is performed on the incoming particles whereas $\Delta M\left(\mathbf{v}_{1}, \mathbf{v}_{2}, \mathbf{b}\right)$ is evaluated in terms of the outgoing particles $(+)$ and therefore must be identified with the second equation on the rhs of (40). Consider now the dependence in terms of the outgoing particle velocities $\mathbf{v}_{1}^{(+)}$and $\mathbf{v}_{2}^{(+)}$in the previous phase-space integral. The velocity dependence contained in the factors $\left|\mathbf{v}_{12} \cdot \mathbf{n}_{12}\right|$ and $\partial \widehat{\rho}_{1}^{(N)}\left(\mathbf{r}_{1}, \mathbf{v}_{1}^{(+)}, t\right) / \partial \mathbf{r}_{1} \cdot \partial \hat{\rho}_{1}^{(N)}\left(\mathbf{r}_{2}, \mathbf{v}_{2}^{(+)} t\right) / \partial \mathbf{r}_{2}$ is symmetric with respect to the variables $\mathbf{v}_{1}^{(+)}$and $\mathbf{v}_{2}^{(+)}$. On the other hand, as a whole, the same integral should remain unaffected with respect to the exchange of the outgoing particle velocities $\mathbf{v}_{1}^{(+)} \Longleftrightarrow \mathbf{v}_{2}^{(+)}$. This means that the only term in $\Delta M\left(\mathbf{v}_{1}, \mathbf{v}_{2}, \mathbf{b}\right)$ which gives a (possibly) nonvanishing contribution is $-(\mathbf{b}$. $\left.\mathbf{n}_{12}\right)^{2}\left(\mathbf{n}_{12} \cdot \mathbf{v}_{12}^{(+)}\right)^{2}$. As a consequence it is found that

$$
\begin{aligned}
& \frac{\partial}{\partial t} K_{M}\left(\rho_{1}^{(N)}(t), \mathbf{b}\right) \equiv W_{M}\left(\rho_{1}^{(N)}(t), \mathbf{b}\right)=-(N-1) \\
& \cdot \sigma^{2} \int_{U_{1(1)}} d \mathbf{v}_{1} \int_{U_{1(2)}} d \mathbf{v}_{2} \times \int_{\Omega} d \mathbf{r}_{1} \bar{\Theta}_{1}^{(\partial \Omega)}\left(\overline{\mathbf{r}}_{1}\right) \\
& \quad \cdot \int^{(-)} d \mathbf{\Sigma}_{21} \frac{\partial \widehat{\rho}_{1}^{(N)}\left(\mathbf{r}_{1}, \mathbf{v}_{1}^{(+)}, t\right)}{\partial \mathbf{r}_{1}} \\
& \quad \cdot \frac{\partial \widehat{\rho}_{1}^{(N)}\left(\mathbf{r}_{2}=\mathbf{r}_{1}+\sigma \mathbf{n}_{21}, \mathbf{v}_{2}^{(+)}, t\right)}{\partial \mathbf{r}_{2}} \times\left|\mathbf{v}_{12}^{(+)} \cdot \mathbf{n}_{12}\right| \\
& \quad \cdot\left(\mathbf{b} \cdot \mathbf{n}_{12}\right)^{2}\left(\mathbf{n}_{12} \cdot \mathbf{v}_{12}^{(+)}\right)^{2} k_{2}^{(N)}\left(\mathbf{r}_{1}, \mathbf{r}_{2}=\mathbf{r}_{1}+\sigma \mathbf{n}_{21}, t\right) \\
& \leq 0
\end{aligned}
$$

and hence $(\partial / \partial t) K_{M}\left(\rho_{1}^{(N)}(t), \mathbf{b}\right)$ is necessarily negative or null, the second case occurring only if $\partial \widehat{\rho}_{1}^{(N)}\left(\mathbf{r}_{1}, \mathbf{v}_{1}^{(+)}, t\right) / \partial \mathbf{r}_{1} \equiv$ 0 and consequently $\partial \hat{\rho}_{1}^{(N)}\left(\mathbf{r}_{2}=\mathbf{r}_{1}+\sigma \mathbf{n}_{21}, \mathbf{v}_{2}^{(+)} t\right) / \partial \mathbf{r}_{2} \equiv 0$ too.

The proof of Proposition $\mathrm{P} 2{ }_{2}$ follows in a similar way. In fact, first, one notices that thanks to the global validity of the 1-body PDF [13] the 1-body PDF $\rho_{1}^{(N)}(t)$ necessarily belongs to the functional class of stochastic PDFs $\left\{\rho_{1}^{(N)}\left(\mathbf{x}_{1}, t\right)\right\}$ prescribed so that also the local characteristic scale length defined above $L_{\rho}(t)$ (see (16)) is larger than zero and finite. As a consequence it follows that both the functional $K_{M}\left(\rho_{1}^{(N)}(t), \mathbf{b}\right)$ and $I_{M}\left(\rho_{1}^{(N)}(t), \mathbf{b}\right)$ (see $\left.(22)\right)$ are globally defined too. Consider in fact the representation of $K_{M}\left(\rho_{1}^{(N)}(t), \mathbf{b}\right)$ achieved in Theorem 1 and given by (35). Next, let us notice that thanks to (16) the characteristic scale length

$$
L_{\mu, \min } \equiv \inf \left\{\left|\frac{\partial \hat{\rho}_{1}^{(N)}\left(\mathbf{x}_{1}, t\right)}{\partial \mathbf{r}_{1}}\right|^{-1}\right\}
$$

is necessarily strictly positive. Then, upon noting that $k_{2}^{(N)}\left(\mathbf{r}_{1}\right.$, $\left.\mathbf{r}_{2}, t\right) \leq 1$ and $\int_{\Gamma_{1(2)}} d \mathbf{x}_{2} \delta\left(\left|\mathbf{r}_{2}-\mathbf{r}_{1}\right|-\sigma\right) \widehat{\rho}_{1}^{(N)}\left(\mathbf{x}_{2}, t\right) \leq \sup \left(\widehat{n}_{1}^{(N)}\left(\mathbf{r}_{2}\right.\right.$, $t))<+\infty$, with $\widehat{n}_{1}^{(N)}\left(\mathbf{r}_{2}, t\right)$ being the velocity moment $\widehat{n}_{1}^{(N)}\left(\mathbf{r}_{2}\right.$, $t)=\int_{U_{1(2)}} d \mathbf{v}_{2} \widehat{\rho}_{1}^{(N)}\left(\mathbf{r}_{2}, \mathbf{v}_{2}, t\right)$, it follows that

$$
\begin{aligned}
K_{M}\left(\rho_{1}^{(N)}(t), \mathbf{b}\right) \leq & \frac{(N-1)}{L_{\mu, \min }} \sup \left(\widehat{n}_{1}^{(N)}\left(\mathbf{r}_{2}, t\right)\right) \\
& \cdot \int_{\Gamma_{1(1)}} d \mathbf{x}_{1} \bar{\Theta}_{1}^{(\partial \Omega)}\left(\overline{\mathbf{r}}_{1}\right) M\left(\mathbf{v}_{1}, \mathbf{b}\right) \\
& \cdot \hat{\rho}_{1}^{(N)}\left(\mathbf{x}_{1}, t\right)
\end{aligned}
$$

where the integral on the rhs is necessarily bounded. This happens because $\rho_{1}^{(N)}\left(\mathbf{x}_{1}, t\right)$ belongs to the functional class $\left\{\rho_{1}^{(N)}(t)\right\}$ and therefore $\widehat{n}_{1}^{(N)}\left(\mathbf{r}_{2}, t\right)$ is bounded, while, at the same time, the phase-space moments indicated above necessarily exist. Furthermore, since $(\partial / \partial t) I_{M}\left(\rho_{1}^{(N)}(t), \mathbf{b}\right) \equiv$ $\left(1 / K_{M o}\right)(\partial / \partial t) K_{M}\left(\rho_{1}^{(N)}(t), \mathbf{b}\right)$, the inequality (53) implies (42) and (43) too. Finally, since $\hat{\rho}_{1}^{(N)}\left(\mathbf{r}_{1}, \mathbf{v}_{1}, t\right)$ is a solution of the Master kinetic equation $\left(\partial / \partial \mathbf{r}_{1}\right) \hat{\rho}_{1}^{(N)}\left(\mathbf{x}_{1}, t\right) \equiv 0$ occurs if and only if $\hat{\rho}_{1}^{(N)}\left(\mathbf{x}_{1}, t\right)$ coincides with a Maxwellian kinetic equilibrium of the type (6). This result proves therefore also Proposition $\mathrm{P} 2_{3}$.

The implication of Theorem 2 is therefore that provided the initial value $K_{M}\left(\rho_{1 o}^{(N)}\left(\mathbf{x}_{1}\right), \mathbf{b}\right)$ is nonvanishing then necessarily:

(i) the functional $K_{M}\left(\rho_{1}^{(N)}(t), \mathbf{b}\right)$ is monotonically decreasing and thus $K_{M}\left(\rho_{1}^{(N)}(t), \mathbf{b}\right) \leq K_{M}\left(\rho_{1 o}^{(N)}\left(\mathbf{x}_{1}\right), \mathbf{b}\right)$;

(ii) similarly the MKI functional $I_{M}\left(\rho_{1}^{(N)}(t), \mathbf{b}\right)$ is monotonically decreasing too, i.e., $I_{M}\left(\rho_{1}^{(N)}(t), \mathbf{b}\right) \leq$ $I_{M}\left(\rho_{1 o}^{(N)}\left(\mathbf{x}_{1}\right), \mathbf{b}\right)$;

(iii) both $K_{M}\left(\rho_{1}^{(N)}(t), \mathbf{b}\right)$ and $I_{M}\left(\rho_{1}^{(N)}(t), \mathbf{b}\right)$ are nonnegative.

2.3. Proof of the DKE Property for the Master Kinetic Equation. Let us now show that in validity of Theorems 1 and 2 the timeevolved $\rho_{1}^{(N)}\left(\mathbf{x}_{1}, t\right)$ necessarily must decay asymptotically for $\tau \equiv t-t_{o} \longrightarrow+\infty$ to kinetic equilibrium, i.e., that the limit function $\lim _{\tau \longrightarrow+\infty} \rho_{1}^{(N)}\left(\mathbf{x}_{1}, t\right) \equiv \rho_{1 \infty}^{(N)}\left(\mathbf{x}_{1}\right)$ exists and it necessarily coincides with a Maxwellian kinetic equilibrium of the type (6). In this regard the following proposition holds.

Theorem 3 (asymptotic behavior of $I_{M}\left(\rho_{1}^{(N)}(t), \mathbf{b}\right)$ (master equation-DKE theorem)). Let us assume that the initial condition $\rho_{1 o}^{(N)}\left(\mathbf{x}_{1}\right) \in\left\{\rho_{1 o}^{(N)}\left(\mathbf{x}_{1}\right)\right\}$ is such that the corresponding functional $K_{M}\left(\rho_{1 o}^{(N)}\left(\mathbf{x}_{1}\right), \mathbf{b}\right)$ is nonvanishing, i.e., in view of Theorem 1 necessarily $>0$. Then it follows that the corresponding time-evolved solution of the Master kinetic equation $\rho_{1}^{(N)}\left(\mathbf{x}_{1}, t\right)$ in the limit $\tau \equiv t-t_{o} \longrightarrow+\infty$ necessarily must decay to kinetic equilibrium, i.e.,

$$
\lim _{\tau \longrightarrow+\infty} \rho_{1}^{(N)}\left(\mathbf{x}_{1}, t\right)=\rho_{1 M}^{(N)}\left(\mathbf{v}_{1}\right) .
$$


Proof. In order to reach the thesis it is sufficient to prove that necessarily

$$
\lim _{\tau \longrightarrow+\infty} \frac{\partial}{\partial t} I_{M}\left(\rho_{1}^{(N)}(t), \mathbf{b}\right)=0 .
$$

In fact, let us assume "ad absurdum" that $(\partial / \partial t) I_{M}\left(\rho_{1}^{(N)}(t)\right.$, b) $\leq-k^{2}$ with $k^{2}>0$ being a real constant. Then Theorem 2 (Proposition $\mathrm{P} 2_{2}$ ) requires that

$$
\lim _{\tau \longrightarrow+\infty} I_{M}\left(\rho_{1}^{(N)}(t), \mathbf{b}\right) \leq-\lim _{\tau \longrightarrow+\infty}\left(t-t_{o}\right) k^{2}=-\infty,
$$

a result which contradicts Theorem 1 . This proves the validity of (57). Furthermore, by construction $(\partial / \partial t) I_{M} \equiv$ $\left(1 / K_{M o}\right)(\partial / \partial t) K_{M}$ and furthermore $(\partial / \partial t) K_{M}$ is identified with the functional $W_{M}\left(\rho_{1}^{(N)}(t), \mathbf{b}\right) \leq 0$ which is determined by (53). At this point one notices that, thanks to continuity of the functional $W_{M}\left(\rho_{1}^{(N)}(t), \mathbf{b}\right)$, the identity

$$
\lim _{\tau \longrightarrow+\infty} \frac{\partial}{\partial t} I_{M}\left(\rho_{1}^{(N)}\left(\mathbf{x}_{1}, t\right), \mathbf{b}\right)=W_{M}\left(\rho_{1 \infty}^{(N)}\left(\mathbf{x}_{1}\right), \mathbf{b}\right)
$$

holds, where, thanks to global existence of the 1-body PDF (see [13]), the limit function

$$
\lim _{\tau \longrightarrow+\infty} \rho_{1}^{(N)}\left(\mathbf{x}_{1}, t\right) \equiv \rho_{1 \infty}^{(N)}\left(\mathbf{x}_{1}\right)
$$

necessarily exists. As a consequence (57) requires also the equation

$$
W_{M}\left(\rho_{1 \infty}^{(N)}\left(\mathbf{x}_{1}\right), \mathbf{b}\right)=0
$$

to hold. Upon invoking Proposition $\mathrm{P} 2_{3}$ of Theorem 2 this implies that necessarily $\rho_{1 \infty}^{(N)}\left(\mathbf{x}_{1}\right)=\rho_{1 M}^{(N)}\left(\mathbf{v}_{1}\right)$ so the thesis (56) is proved. Incidentally, thanks to Theorem 1 , this requires also that

$$
\lim _{\tau \longrightarrow+\infty} I_{M}\left(\rho_{1}^{(N)}(t), \mathbf{b}\right)=I_{M}\left(\rho_{1 \infty}^{(N)}\left(\mathbf{x}_{1}\right), \mathbf{b}\right)=0 .
$$

2.4. Remarks. A few remarks are worth being pointed out regarding the results presented above.

(1) Remark \#1. The choice of the MKI functional considered here (see (22)) is just one of the infinite particular admissible realizations which meet the complete set of MKI prescriptions indicated above. In particular, the choice of the velocity moment $M\left(\mathbf{v}_{1}, \mathbf{b}\right)$ considered here (see (24)) remains in principle arbitrary, since $\left|\mathbf{v}_{1} \cdot \mathbf{b}\right|^{2}$ can be equivalently replaced, for example, by any factor of the form $\left|\mathbf{v}_{1} \cdot \mathbf{b}\right|^{2 n}$, with $n \geq 1$. Furthermore it is obvious that $M\left(\mathbf{v}_{1}, \mathbf{b}\right)$ can be replaced by any function of the form $M\left(\mathbf{v}_{1}, \mathbf{b}\right)+\Delta M\left(\mathbf{v}_{1}, \mathbf{b}\right)$, being $\Delta M\left(\mathbf{v}_{1}, \mathbf{b}\right)$ prescribed in such a way that its contribution to $(\partial / \partial t) I_{M}$ vanishes identically so that the validity of the inequality (42) in Theorem 2 is preserved. This implies in turn that the prescription of the MKI functional $I_{M}\left(\rho_{1}^{(N)}(t)\right)$ remains in principle nonunique.
(2) Remark \#2. A possible issue is related to the requirement that the renormalized 1-body PDF, as the 1-body PDF itself, are strictly positive at all times and are nonvanishing. Here it is sufficient to state that an elementary consequence of the theory of the Master kinetic equation developed in [3] is that, provided the corresponding initial $N$-body PDF set at a prescribed initial time $t_{o}$ is strictly positive in the whole $N$-body phase-space, both the corresponding renormalized 1 -body PDF as the 1-body PDF remain necessarily strictly positive globally in time too and everywhere in the 1-body phase-space.

(3) Remark \#3. It must be stressed that the signature of the time derivative $(\partial / \partial t) I_{M}\left(\rho_{1}^{(N)}(t), \mathbf{b}\right)$ actually depends crucially on the adoption of the causal form of MCBC (i.e., see (C.1) or (C.3) in Appendix C) rather than the anticausal one (given instead by (C.2)). The first choice is mandatory in view of the causality principle. Indeed, it is immediate to prove that $(\partial /$ $\partial t) I_{M}\left(\rho_{1}^{(N)}(t), \mathbf{b}\right)$ changes signature if the anticausal MCBC (C.2) is invoked.

(4) Remark \#4. Theorem 2 warrants that macroscopic irreversibility, namely, the inequality $(\partial / \partial t) I_{M}\left(\rho_{1}^{(N)}(t)\right.$, b) $\leq 0$, occurs specifically because of (a) the timevariation of the $\mathbf{b}$-directional total kinetic energy which occurs at arbitrary binary-collision events; (b) the occurrence of a velocity-space anisotropy in the 1-body PDF, i.e., the fact that the same PDF may not coincide with a local Maxwellian PDF.

(5) Remark \#5. The existence of the limit function $\lim _{t \longrightarrow+\infty} \rho_{1}^{(N)}\left(\mathbf{x}_{1}, t\right)=\rho_{1 \infty}^{(N)}\left(\mathbf{x}_{1}\right)$ follows uniquely as a consequence of the global existence theorem holding for the Master kinetic equation [13].

(6) Remark \#6. Last but not least, the fact that the same limit function may coincide or not with the Maxwellian kinetic equilibrium (6) depends crucially on the functional setting prescribed for the same PDF $\rho_{1}^{(N)}\left(\mathbf{x}_{1}, t\right)$. More precisely, DKE can only occur provided $\rho_{1}^{(N)}\left(\mathbf{x}_{1}, t\right)$ is a suitably smooth stochastic PDF such that the MKI functional exists for the corresponding initial PDF at time $t_{o}$, i.e., $\rho_{1}^{(N)}\left(\mathbf{x}_{1}, t_{o}\right)=$ $\rho_{1 o}^{(N)}\left(\mathbf{x}_{1}\right)$.

Theorems 1-3 represent the main results reached in the paper of what may be referred to as the PMI/DKE theory for finite hard-sphere systems and which have concerned the axiomatic formulation in such a context of the notion of macroscopic irreversibility and the related one of decay to kinetic equilibrium.

\section{Consistency of MPI/DKE Theory with Microscopic Dynamics}

The crucial problem which arises in the context of the ab initio theory is in some sense analogous to that occurring in the Boltzmann and Grad kinetic theories. The question is in fact whether these phenomena are actually consistent 
with the fundamental symmetry properties of the underlying Boltzmann-Sinai CDS. The problem posed in the present section concerns, more precisely, the consistency with the time-reversible, energy-conserving, evolution of the underlying $N$-body Boltzmann-Sinai classical dynamical system $S_{N}-$ CDS.

(1) First issue: consistency with the microscopic reversibility principle. This is related to the famous objection raised by Loschmidt to the Boltzmann equation and Boltzmann H-theorem: i.e., whether and possibly also how it may be possible to reconcile the validity of the reversibility principle for the $S_{N}$-CDS with the manifestation of a decay of the 1-body PDF to kinetic equilibrium, i.e., the uniform Maxwellian PDF of the form (6), as predicted by the above Master equation-DKE Theorem. That a satisfactory answer to this question is actually possible follows from considerations which are based on the axiomatic (ab initio) statistical description realized by the Master kinetic equation. In this regard it is worth recalling the discussion reported above concerning the role of MCBC regarding the functional $(\partial / \partial t) I_{M}\left(\rho_{1}^{(N)}(t)\right)$. In particular, it is obvious that the signature depends on whether the causal (or anticausal) form of MCBC is invoked (see Appendix C). Such a choice is not arbitrary since, for consistency with the causality principle, it must depend on the microscopic arrow of time, i.e., the orientation of the time axis chosen for the reference frame. Based on these premises, consistency between the occurrence of macroscopic irreversibility associated with the DKE phenomenon and the principle of microscopic reversibility can immediately be established. Indeed, it is sufficient to notice that when a time-reversal or a velocityreversal is performed on the $S_{N}$-CDS the form of the collision boundary conditions (i.e., in the present case the MCBC provided by (C.1) in Appendix C) must be changed, replacing them with the corresponding anticausal ones, i.e., (C.2). This implies that MKI functional decreases in both cases, i.e., after performing the time-reversal, so that no contradiction can possibly arise in this case between Theorem 3 and the microscopic reversibility principle.

(2) Second issue: consistency with Poincaré recurrence theorem. Similar considerations concern the consistency with the recurrence theorem due to Poincaré as well as the conservation of total (kinetic) energy for the $S_{N}-$ CDS. In fact, first, as it follows from [3], by construction the Master collision operator admits the customary Boltzmann collisional invariants, including total kinetic energy of colliding particles. Hence, total energy conservation is again warranted for $S_{N}-\mathrm{CDS}$. Second, regarding Poincaré recurrence theorem, it concerns the Lagrangian phase-space trajectories of the $S_{N}-\mathrm{CDS}$, i.e., the fact that almost all of these trajectories return arbitrarily close-in a suitable sense to be prescribed in terms of a distance defined on the $\mathrm{N}$-body phase-space-to their initial condition after a suitably large "recurrence time". Incidentally, its magnitude depends strongly both on the same initial condition and the notion of distance to be established on the same phase-space. Nevertheless, such a "recurrence effect" influences only the Lagrangian time evolution of the $N$-body PDF which occurs along the same Lagrangian $\mathrm{N}$-body phasespace trajectories. Instead, the same recurrence effect has manifestly no influence on the time evolution of the Eulerian 1-body PDF which is advanced in time in terms of the Eulerian kinetic equation represented by the Master kinetic equation. Therefore the mutual consistency of DKE and Poincaré recurrence theorem remains obvious.

Hence, in the framework of the axiomatic ab initio theory based on the Master kinetic equation the full consistency is warranted with the microscopic dynamics of the underlying Boltzmann-Sinai CDS.

\section{Physical Implications}

Let us now investigate the physical interpretation and main implications emerging from the PMI/DKE theory developed here. The first issue is related to the physical mechanism at the basis of the PMI/DKE phenomenology.

It is well known that in the context of Boltzmann kinetic theory the property of macroscopic irreversibility and the occurrence of the DKE phenomenon are both ascribed to the Boltzmann H-theorem, both in its original formulation [7] and in its modified form introduced by Boltzmann himself while attempting to reply [28] to Loschmidt objection [27] (see also $[30,31]$ together with different views on the matter given in $[24,26])$. As recalled above, this is expressed in terms of the production rate for the Boltzmann-Shannon entropy $(\partial / \partial t) S\left(\rho_{1}(t)\right)$, with $S\left(\rho_{1}(t)\right)$ being interpreted as a measure of the ignorance associated with a solution of the Boltzmann equation. In fact the customary interpretation is that they arise specifically because of the validity of the entropic inequality (10), i.e., the monotonic increase of $S\left(\rho_{1}(t)\right)$, and the corresponding entropic equality (11) stating a necessary and sufficient condition for kinetic equilibrium. Such a theorem is actually intimately related to the equation itself. In fact both the theorem and the equation generally hold only for stochastic PDFs $\rho_{1}(t)=\rho_{1}\left(\mathbf{x}_{1}, t\right)$ which are suitably smooth and not for distributions [1]. According to Boltzmann's original interpretation, however, both the Boltzmann equation and Boltzmann $\mathrm{H}$-theorem should only hold when the so-called Boltzmann-Grad limit is invoked, i.e., based on the limit operator $L_{B G} \equiv \lim _{N \rightarrow+\infty}$ (see [4]). $N \sigma^{2} \sim \mathrm{O}(1)$

In striking departure from such a picture, one has the following:

(i) The axiomatic ab initio theory based on the Master kinetic equation and the present PMI/DKE theory are applicable to an arbitrary finite Boltzmann-Sinai CDS. This means that they hold for hard-sphere systems having a finite number of particles and with 
finite diameter and mass, i.e., without the need of invoking validity of asymptotic conditions.

(ii) The main departure with respect to Boltzmann kinetic theory arises because, as earlier discovered (see in particular the related discussion reported in [4]), the Boltzmann-Shannon entropy associated with an arbitrary stochastic 1-body $\operatorname{PDF} \rho_{1}^{(N)}(t)=$ $\rho_{1}^{(N)}\left(\mathbf{x}_{1}, t\right)$ solution of the Master kinetic equation is identically conserved. Thus both PMI and DKE are essentially unrelated to the Boltzmann-Shannon entropy.

(iii) In the case of the Master kinetic equation the physical mechanism responsible for the occurrence of both PMI and DKE is unrelated to the BoltzmannShannon entropy. In fact, as shown here, it arises because of the properties of the MKI functional $I_{M}\left(\rho_{1}^{(N)}(t), \mathbf{b}\right)$ when it is expressed in terms of an arbitrary stochastic $\operatorname{PDF} \rho_{1}^{(N)}(t)=\rho_{1}^{(N)}\left(\mathbf{x}_{1}, t\right)$ solution of the Master kinetic equation. The only requirement is that the initial PDF $\rho_{1 o}^{(N)}\left(\mathbf{x}_{1}\right)$ is prescribed so that the corresponding MKI functional $I_{M}\left(\rho_{1 o}^{(N)}\left(\mathbf{x}_{1}\right), \mathbf{b}\right)$ exists.

(iv) As shown here the MKI functional is a suitably weighted phase-space moment of $\rho_{1}^{(N)}\left(\mathbf{x}_{1}, t\right)$ which can be interpreted as an information measure for the same PDF, namely, belongs to the interval $[0,1]$, and exhibits a monotonic-decreasing time-dependence, i.e., the property of macroscopic irreversibility.

(v) In addition both $I_{M}\left(\rho_{1}^{(N)}(t), \mathbf{b}\right)$ and its time derivative $(\partial / \partial t) I_{M}\left(\rho_{1}^{(N)}(t), \mathbf{b}\right)$ vanish identically if and only if the 1-body PDF coincides with a Maxwellian kinetic equilibrium of the type (6). This warrants in turn also the occurrence of the DKE phenomenon for $\rho_{1}^{(N)}\left(\mathbf{x}_{1}, t\right)$, i.e., that for $t-t_{o} \longrightarrow+\infty$ the same PDF must decay to a Maxwellian kinetic equilibrium of this type.

(vi) Finally, it is interesting to point out the peculiar behavior of the MKI functional $I_{M}\left(\rho_{1}^{(N)}(t), \mathbf{b}\right)$ and its time derivative $(\partial / \partial t) I_{M}\left(\rho_{1}^{(N)}(t), \mathbf{b}\right)$ when the Boltzmann-Grad limit is considered. In particular the 1- and 2-body occupation coefficients $k_{1}^{(N)}\left(\mathbf{r}_{1}, t\right)$ and $k_{2}^{(N)}\left(\mathbf{r}_{1}, \mathbf{r}_{2}, t\right)$ which appear in the Master kinetic equation (see Appendix B, (B.1) and (B.2)) become respectively

$$
\begin{aligned}
L_{B G} k_{1}^{(N)}\left(\mathbf{r}_{1}, t\right) & =1 \\
L_{B G} k_{2}^{(N)}\left(\mathbf{r}_{1}, \mathbf{r}_{2}, t\right) & =1 .
\end{aligned}
$$

As a consequence the limit functionals $L_{B G} I_{M}\left(\rho_{1}^{(N)}(t), \mathbf{b}\right) \quad$ and $\quad L_{B G}(\partial / \partial t) I_{M}\left(\rho_{1}^{(N)}(t), \mathbf{b}\right)$, are necessarily identically vanishing. This means that the present theory applies properly when the exact Master kinetic equation is considered and not to its asymptotic approximation obtained in the Boltzmann-Grad limit, namely the Boltzmann kinetic equation (see $[3,13])$.

An interesting issue, in the context of the PMI/DKE theory for the Master kinetic equation, is the role of MCBC in giving rise to the phenomena of macroscopic irreversibility and decay to kinetic equilibrium. Let us analyze for this purpose the two cases represented by unary and binary hardsphere elastic collisions.

First, let us recall the customary treatment of collision boundary conditions for unary collision events (also referred to as the so-called mirror reflection CBC; see for example Cercignani $[6,11])$. This refers to the occurrence at a collision time $t_{i}$ of a single unary elastic collision for particle 1 at the boundary $\partial \Omega$. Let us denote by $\mathbf{n}_{1}$ the inward normal to the stationary rigid boundary $\partial \Omega$ at the point of contact with the same particle and respectively $\mathbf{x}_{1}^{(-)}\left(t_{1}\right)=\left(\mathbf{r}_{1}\left(t_{1}\right), \mathbf{v}_{1}^{(-)}\left(t_{1}\right)\right)$ and $\mathbf{x}_{1}^{(+)}\left(t_{1}\right)=\left(\mathbf{r}_{1}\left(t_{1}\right), \mathbf{v}_{1}^{(+)}\left(t_{1}\right)\right)$ the incoming and outgoing particle states while $\mathbf{v}_{1}^{(+)}$is determined by the elastic collision law for unary collisions, namely,

$$
\mathbf{v}_{1}^{(+)}=\mathbf{v}_{1}^{(-)}-2 \mathbf{n}_{1} \mathbf{n}_{1} \cdot \mathbf{v}_{1}^{(-)}
$$

Then, the PDF-conserving CBC for the 1-body PDF requires that the following identity holds:

$$
\rho_{1}^{(N)}\left(\mathbf{x}_{1}^{(+)}\left(t_{1}\right), t_{i}\right)=\rho^{(N)}\left(\mathbf{x}_{1}^{(-)}\left(t_{i}\right), t_{i}\right),
$$

with $\rho_{1}^{(N)}\left(\mathbf{x}_{1}^{(+)}\left(t_{1}\right), t_{i}\right) \equiv \rho_{1}^{(N)(+)}\left(\mathbf{x}_{1}^{(+)}\left(t_{1}\right), t_{i}\right)$ and $\rho^{(N)}\left(\mathbf{x}_{1}^{(-)}\left(t_{i}\right)\right.$, $\left.t_{i}\right) \equiv \rho^{(N)(-)}\left(\mathbf{x}_{1}^{(-)}\left(t_{i}\right), t_{i}\right)$ denoting the outgoing and incoming 1 -body PDF, respectively. This identifies the PDF-conserving CBC usually adopted in Boltzmann kinetic theory [7] (Grad $[10]$; see also $[2,3])$. The obvious physical implication of (65) is that $\rho_{1}^{(N)}\left(\mathbf{x}_{1}^{(+)}\left(t_{1}\right), t_{i}\right)$ (and $\left.\rho^{(N)}\left(\mathbf{x}_{1}^{(-)}\left(t_{i}\right), t_{i}\right)\right)$ should be necessarily an even function of the velocity component $\mathbf{n}_{1}$. $\mathbf{v}_{1}^{(-)}$. Indeed as shown in $[2,3]$ the PDF-conserving CBC (65) should be replaced with a suitable $\mathrm{CBC}$ identified with the MCBC condition (see also Appendix C). When realized in terms of its causal form (predicting the outgoing PDF in terms of the incoming one) the MCBC for unary collisions is just

$$
\rho_{1}^{(N)(+)}\left(\mathbf{x}_{1}^{(+)}\left(t_{1}\right), t_{i}\right)=\rho^{(N)(-)}\left(\mathbf{x}_{1}^{(+)}\left(t_{i}\right), t_{i}\right),
$$

with $\rho^{(N)(-)}\left(\mathbf{x}_{1}^{(+)}\left(t_{i}\right), t_{i}\right)$ denoting the incoming 1-body PDF evaluated in terms of the outgoing state $\mathbf{x}_{1}^{(+)}\left(t_{i}\right)$. Assuming left-continuity (see related discussion in [2]), this can then be identified with $\rho^{(N)(-)}\left(\mathbf{x}_{1}^{(+)}\left(t_{i}\right), t_{i}\right) \equiv \rho^{(N)}\left(\mathbf{x}_{1}^{(+)}\left(t_{i}\right), t_{i}\right)$, thus yielding

$$
\rho_{1}^{(N)(+)}\left(\mathbf{x}_{1}^{(+)}\left(t_{1}\right), t_{i}\right)=\rho^{(N)}\left(\mathbf{x}_{1}^{(+)}\left(t_{i}\right), t_{i}\right) .
$$

Equation (67) provides the physical prescription for the collision boundary condition, which is referred to as MCBC, holding for the 1-body PDF at arbitrary unary collision events. It is immediate to realize that the function $\rho^{(N)}\left(\mathbf{x}_{1}^{(+)}\left(t_{i}\right), t_{i}\right)$ needs not generally be even with respect to the velocity component $\mathbf{n}_{1} \cdot \mathbf{v}_{1}^{(-)}$. In addition (67), just as 
(65), also permits the existence of the customary collisional invariants which in the case of unary collisions are $X=$ $1,\left|\mathbf{n}_{1} \cdot \mathbf{v}_{1}^{(-)}\right|, \mathbf{v}_{1} \cdot\left[\underline{\underline{1}}-\mathbf{n}_{1} \mathbf{n}_{1}\right], v_{1}^{2}$. As a consequence, one can show that (67) warrants at the same time also the validity of the socalled no-slip boundary conditions for the fluid velocity field $\mathbf{V}\left(\mathbf{r}_{1}, t\right)$ carried by the 1 -body $\operatorname{PDF} \rho_{1}^{(N)}\left(\mathbf{x}_{1}, t\right)$.

The treatment of MCBC holding for the 2-body PDF in case of binary-collision events is analogous and is recalled for convenience in (C.2) of Appendix C.

Let us briefly analyze the qualitative physical implications of (67) and (C.2) as far as the DKE theory is concerned. First, we notice that unary collisions cannot produce in a proper sense a velocity-isotropization effect since, as shown by (67), in such a case MCBC gives rise only to a change in the velocity distribution occurring during a unary collision due to a single component of the particle velocity, namely, $\mathbf{n}_{1} \cdot \mathbf{v}_{1}^{(-)}$. As a consequence, this explains why unary collisions do not affect the rate of change of the MKI functional (see Theorem 2). Second, (C.2) shows-on the contrary - that binary collisions actually do affect by means of MCBC a velocity-spreading for the 1- and 2-body PDF. In particular, since the spreading effect occurs in principle for all components of particle velocities affecting both particles 1 and 2, this explains why binary collisions are actually responsible for the irreversible time evolution of the MKI functional (see Theorems 2 and 3).

In turn, as implied by Theorem 3, DKE arises because of the phenomenon of macroscopic irreversibility (Theorem 2). The latter arises due specifically to the possible occurrence of a velocity-space anisotropy which characterizes the 1-body PDF when the same PDF differs locally from kinetic equilibrium. In turn, this requires also that the 1-body PDF belongs to the functional class of admissible stochastic PDFs $\left\{\rho_{1}^{(N)}\left(\mathbf{x}_{1}, t\right)\right\}$. In difference to Boltzmann kinetic theory, however, the key physical role is actually ascribed to the MKI functional $I_{M}\left(\rho_{1}^{(N)}(t)\right)$ rather than the BoltzmannShannon entropy $S_{1}\left(\rho_{1}^{(N)}(t)\right)$. In fact, recalled above, the same functional remains constant in time once the Master kinetic equation is adopted. Rather, as shown by Theorem 2, it is actually the Master kinetic information $I_{M}\left(\rho_{1}^{(N)}(t)\right)$ which exhibits the characteristic signatures of macroscopic irreversibility.

The key differences arising between the two theories, i.e., the Boltzmann equation-DKE and the Master equationDKE, are of course related to the different and peculiar intrinsic properties of the Boltzmann and Master kinetic equations. In particular, as discussed at length elsewhere (see $[1,3])$, precisely because the Boltzmann equation is only an asymptotic approximation of the Master kinetic equation explains why a loss of information occurs in Boltzmann kinetic theory and consequently the related BoltzmannShannon entropy is not conserved.

The present investigation shows that in the context of the Master kinetic equation, the macroscopic irreversibility property, i.e., the monotonic time-decay behavior of the MKI functional, can be explained at a more fundamental level, i.e., based specifically on the time-variation of the $\mathbf{b}$-directional total kinetic energy which occurs at arbitrary binary-collision events.
The Master equation-DKE theorem (Theorem 3) given above provides a first-principle proof of the existence of the phenomenon of DKE occurring for the kinetic description of a finite number of extended hard-spheres, i.e., described by means of the Master kinetic equation. More precisely, the DKE phenomenon affects the 1 -body PDFs belonging to the admissible functional class $\left\{\rho_{1}^{(N)}\left(\mathbf{x}_{1}, t\right)\right\}$ determined according to Theorem 1 and requiring also that the local characteristic scale length $L_{\rho}(t)$ associated with $\rho_{1}^{(N)}\left(\mathbf{x}_{1}, t\right)$ is nonzero at all times.

\section{Conclusions}

In this paper the problem of the property of microscopic irreversibility (PMI) and decay to kinetic equilibrium (DKE) of the 1-body PDF has been addressed. In doing so original ideas and methods are adopted of the new ab initio theory for hard-sphere systems recently developed in the context of classical statistical mechanics $[1,2]$.

These are not just small deviations from standard literature approaches. Such developments, in fact, have opened up a host of exciting new subjects of investigation and theoretical challenges in kinetic theory which arise thanks to, or in the context of, the ab initio approach to kinetic theory. Both are based in particular on the discovery of the Master kinetic equation first reported in [3], equation which has been adopted also in the present paper.

The ab initio theory and specifically the present paper represent the attempt to reach a new foundational basis and axiomatic physical description of the classical statistical mechanics for hard-sphere systems. The topic which has been pursued here-which represents also a challenging test of the $a b$ initio theory itself-concerns the investigation of the physical origins of PMI and the related DKE phenomenon arising in finite $\mathrm{N}$-body hard-sphere systems. These issues refer in particular to

(i) the proof of the nonnegativity of Master kinetic information (Theorem 1, Section 2.1) together with the property of macroscopic irreversibility (PMI; Theorem 2, Section 2.2);

(ii) the establishment of Theorem 3 (Section 2.3) and the related proof of the property of decay to kinetic equilibrium (DKE);

(iii) the consistency of PMI and DKE with microscopic dynamics (Section 3);

(iv) the analysis of the main physical implications of DKE (Section 4).

The theory presented here departs in several respects from previous literature and notably from Boltzmann kinetic theory. The main differences actually arise because of the nonasymptotic character of the new theory, i.e., the fact that it applies to arbitrary dense or rarefied systems for which the finite number and size of the constituent particles is accounted for [3]. In this paper basic consequences of the new theory have been investigated which concern the phenomenon of decay to global kinetic equilibrium. 
The present results are believed to be crucial, besides in mathematical research, for the physical applications of the $\mathrm{ab}$ initio theory statistical theory, i.e., the Master kinetic equation. Indeed, regarding challenging future developments of the theory one should mention among others the following examples of possible (and mutually related) routes worth to be explored. One is related to the investigation of the possible effects due to arbitrarily prescribed, i.e., nonvanishing, initial (binary or multibody) phase-space statistical correlations. As recalled above, in fact, the Master equation is appropriate only when suitably prescribed configuration-space statistical correlations are taken into account. The second goal concerns the investigation of the time-asymptotic properties of the same kinetic equation, for which the present paper may represent a useful basis. The third goal refers to the possible extension of the theory to mixtures formed by hard-spheres of different masses and diameter which possibly undergo both elastic and anelastic collisions. Finally, the fourth one concerns the investigation of hydrodynamic regimes for which a key prerequisite is provided by the DKE theory established here.

\section{Appendix}

\section{A. Realizations of the Master Kinetic Equation}

For completeness we recall here the two equivalent forms of the Master kinetic equation [3]. In terms of the renormalized 1 -body PDF $\hat{\rho}_{1}^{(N)}\left(\mathbf{x}_{1}, t\right)$ (see (26)) the first form of the same equation reads

$$
L_{1(1)} \widehat{\rho}_{1}^{(N)}\left(\mathbf{x}_{1}, t\right)=0,
$$

with $L_{1(1)}=\partial / \partial t+\mathbf{v}_{1} \cdot \partial / \partial \mathbf{r}_{1}$ denoting the 1 -body freestreaming operator. Hence it follows

$$
L_{1(1)} \rho_{1}^{(N)}\left(\mathbf{x}_{1}, t\right)=\hat{\rho}_{1}^{(N)}\left(\mathbf{x}_{1}, t\right) L_{1(1)} k_{1}^{(N)}\left(\mathbf{r}_{1}, t\right),
$$

where explicit evaluation of the rhs the last equation (see also (B.12) below) yields

$$
\begin{aligned}
& \widehat{\rho}_{1}^{(N)}\left(\mathbf{x}_{1}, t\right) L_{1(1)} k_{1}^{(N)}\left(\mathbf{r}_{1}, t\right)=(N-1) \sigma^{2} \int_{U_{1(2)}} d \mathbf{v}_{2} \\
& \cdot \int d \Sigma_{21} \mathbf{v}_{21} \cdot \mathbf{n}_{21} \bar{\Theta}^{*}\left(\mathbf{r}_{2}\right) k_{2}^{(N)}\left(\mathbf{r}_{1}, \mathbf{r}_{2}, t\right) \widehat{\rho}_{1}^{(N)}\left(\mathbf{x}_{1}, t\right) \\
& \cdot \widehat{\rho}_{1}^{(N)}\left(\mathbf{x}_{2}, t\right)
\end{aligned}
$$

with $\bar{\Theta}^{*}\left(\mathbf{r}_{2}\right) \equiv \bar{\Theta}_{i}^{(\partial \Omega)}(\overline{\mathbf{r}})$ and $k_{2}^{(N)}\left(\mathbf{r}_{1}, \mathbf{r}_{2}, t\right)$ being identified with the definitions given respectively by (A.6) and (B.1) in Appendix B. Then, consistent with [3] and upon invoking the causal form of MCBC (see (C.3) in Appendix C) the same equation can be written in the equivalent second form of the Master kinetic equation [3]. The corresponding initial-value problem, taking the form

$$
\begin{aligned}
L_{1(1)} \rho_{1}^{(N)}\left(\mathbf{x}_{1}, t\right)-\mathscr{C}_{1}\left(\rho_{1}^{(N)} \mid \rho_{1}^{(N)}\right) & =0, \\
\rho_{1}^{(N)}\left(\mathbf{x}_{1}, t_{o}\right) & =\rho_{1 o}^{(N)}\left(\mathbf{x}_{1}\right),
\end{aligned}
$$

can be shown to admit a unique global solution [13]. Here the notation is standard [3]. Thus

$$
\begin{aligned}
\mathscr{C}_{1} & \left(\rho_{1}^{(N)} \mid \rho_{1}^{(N)}\right) \equiv(N-1) \sigma^{2} \int_{U_{1(2)}} d \mathbf{v}_{2} \int^{(-)} d \Sigma_{21} \\
\cdot & {\left[\widehat{\rho}_{1}^{(N)}\left(\mathbf{r}_{1}, \mathbf{v}_{1}^{(+)}, t\right) \hat{\rho}_{1}^{(N)}\left(\mathbf{r}_{2}, \mathbf{v}_{2}^{(+)}, t\right)\right.} \\
- & \left.\widehat{\rho}_{1}^{(N)}\left(\mathbf{r}_{1}, \mathbf{v}_{1}, t\right) \hat{\rho}_{1}^{(N)}\left(\mathbf{r}_{2}, \mathbf{v}_{2}, t\right)\right] \times\left|\mathbf{v}_{21} \cdot \mathbf{n}_{21}\right| \\
\cdot & k_{2}^{(N)}\left(\mathbf{r}_{1}, \mathbf{r}_{2}, t\right) \bar{\Theta}^{*}\left(\mathbf{r}_{2}\right)
\end{aligned}
$$

identifies the Master collision operator, while $\rho_{1 o}^{(N)}\left(\mathbf{x}_{1}\right)$ is the initial 1-body PDF which belongs to the functional class $\left\{\rho_{1 o}^{(N)}\left(\mathbf{x}_{1}\right)\right\}$ of stochastic, i.e., strictly positive, smooth ordinary functions, 1-body PDFs. Furthermore, the solidangle integral on the rhs of (A.5) is now evaluated on the subset in which $\mathbf{v}_{12} \cdot \mathbf{n}_{12}<0$, while $\mathbf{r}_{2}$ identifies $\mathbf{r}_{2}=\mathbf{r}_{1}+\sigma \mathbf{n}_{21}$, while $k_{1}^{(N)}\left(\mathbf{r}_{1}, t\right)$ and $k_{2}^{(N)}\left(\mathbf{r}_{1}, \mathbf{r}_{2}, t\right)$ coincide, respectively, with the 1 - and 2-body occupation coefficients [3] and $\bar{\Theta}^{*} \equiv$ $\bar{\Theta}^{*}\left(\mathbf{r}_{i}\right)$ is prescribed by

$$
\bar{\Theta}^{*}\left(\mathbf{r}_{i}\right) \equiv \bar{\Theta}_{i}^{(\partial \Omega)}(\overline{\mathbf{r}}) \equiv \bar{\Theta}\left(\left|\mathbf{r}_{i}-\frac{\sigma}{2} \mathbf{n}_{i}\right|-\frac{\sigma}{2}\right),
$$

with $\bar{\Theta}(x)$ being the strong Heaviside theta function

$$
\bar{\Theta}(x)= \begin{cases}1 & y>0 \\ 0 & y \leq 0 .\end{cases}
$$

Regarding the specific identification of the occupation coefficients (recalled in Appendix B) let us preliminarily recall the notion of $S_{N}$-ensemble strong theta function $\bar{\Theta}^{(N)}$. The latter is prescribed, according to [3], by requiring that

$$
\bar{\Theta}^{(N)}(\overline{\mathbf{r}})=1
$$

for all configuration vectors $\overline{\mathbf{r}} \equiv\left\{\mathbf{r}_{1}, \ldots, \mathbf{r}_{N}\right\}$ belonging to the collisionless subset of $\Omega^{(N)}$. This is identified with the open subset of the $N$-body configuration domain $\Omega^{(N)} \equiv \prod_{i=1, N} \Omega$ in which each of the particles of $S_{N}$ is not in mutual contact with any other particle of $S_{N}$ or with the boundary $\partial \Omega$ of $\Omega$. This means that, at any configuration $\overline{\mathbf{r}}, \bar{\Theta}^{(N)}(\overline{\mathbf{r}})$ can be prescribed as

$$
\bar{\Theta}^{(N)}(\overline{\mathbf{r}}) \equiv \prod_{i=1, N} \bar{\Theta}_{i}(\overline{\mathbf{r}}) \bar{\Theta}_{i}^{(\partial \Omega)}(\overline{\mathbf{r}}) .
$$

Here $\bar{\Theta}_{i}^{(\partial \Omega)}(\overline{\mathbf{r}})$ identifies the $i-$ th particle "boundary" theta function

$$
\bar{\Theta}_{i}^{(\partial \Omega)}(\overline{\mathbf{r}}) \equiv \bar{\Theta}_{i}^{(\partial \Omega)}\left(\overline{\mathbf{r}}_{i}\right)=\bar{\Theta}\left(\left|\mathbf{r}_{i}-\mathbf{r}_{W i}\right|-\frac{\sigma}{2}\right),
$$

with $\mathbf{r}_{W i}=\mathbf{r}_{i}-\rho \mathbf{n}_{i}$ and $\rho \mathbf{n}_{i}$ being the inward vector normal to the boundary belonging to the center of the $i$-th particle having a distance $\rho / 2$ from the same boundary. Furthermore 
$\bar{\Theta}_{i}(\overline{\mathbf{r}})$ is the "binary-collision" theta function. A possible identification of $\bar{\Theta}_{i}(\overline{\mathbf{r}})$ which warrants validity of (A.8) is given by the expression

$$
\bar{\Theta}_{i}(\overline{\mathbf{r}}) \equiv \prod_{\substack{j=1, N ; \\ i<j}} \bar{\Theta}\left(\left|\mathbf{r}_{i}-\mathbf{r}_{j}\right|-\sigma\right),
$$

Namely,

$$
\begin{aligned}
\bar{\Theta}_{i}(\overline{\mathbf{r}}) \equiv \prod_{\substack{j=1, N ; \\
i<j}} \bar{\Theta}_{i j}(\overline{\mathbf{r}}), \\
\bar{\Theta}_{i j}(\overline{\mathbf{r}}) \equiv \bar{\Theta}\left(\left|\mathbf{r}_{i}-\mathbf{r}_{j}\right|-\sigma\right) .
\end{aligned}
$$

However, an equivalent possible prescription of $\bar{\Theta}_{i}(\overline{\mathbf{r}})$ is also provided by the alternative realization obtained letting

$$
\begin{aligned}
& \bar{\Theta}_{i}(\overline{\mathbf{r}}) \equiv \prod_{j=1, N ;} \prod_{\substack{m, n=1, N \\
i<j}} \bar{\Theta}_{i j}^{m n}(\overline{\mathbf{r}}), \\
& \bar{\Theta}_{i j}^{m n}(\overline{\mathbf{r}}) \equiv \bar{\Theta}\left(\left|\mathbf{r}_{i}-\mathbf{r}_{j}\right|-\sigma\right) \\
& \times \bar{\Theta}\left(\left|\mathbf{r}_{i}-\mathbf{r}_{m}\right|+\left|\mathbf{r}_{i}-\mathbf{r}_{n}\right|-2 \sigma\right) \\
& \cdot \bar{\Theta}\left(\left|\mathbf{r}_{m}-\mathbf{r}_{n}\right|+\left|\mathbf{r}_{i}-\mathbf{r}_{m}\right|-2 \sigma\right) .
\end{aligned}
$$

Indeed, in the subset of $\Omega^{(N)}$ in which for all $i=1, N$ the rhs of (A.11) is identically equal to unity, the factor $\prod_{m, n=1, N} \bar{\Theta}\left(\mid \mathbf{r}_{i}-\right.$ $i<m<n$

$\left.\mathbf{r}_{m}|+| \mathbf{r}_{i}-\mathbf{r}_{n} \mid-2 \sigma\right) \bar{\Theta}\left(\left|\mathbf{r}_{m}-\mathbf{r}_{n}\right|+\left|\mathbf{r}_{i}-\mathbf{r}_{m}\right|-2 \sigma\right)$ is necessarily equal to unity too. Incidentally, we notice in fact that the latter factor carries the contributions due to triple collisions which are ruled out in the domain of validity of (A.8).

\section{B. Integral and Differential Identities for the Occupation Coefficients}

One notices that although the definitions (A.13) and (A.11) given in Appendix A for $\bar{\Theta}_{i}(\overline{\mathbf{r}})$ coincide in the collisionless subset of $\Omega^{(N)}$, only the first one is applicable in the complementary collision subset. Based on these premises in this appendix a number of integral and differential identities holding for the 1- and 2-body occupation coefficients are displayed.

First, recalling [3], one notices that the realizations of the 1 - and $s$-body occupation coefficients $k_{1}^{(N)}\left(\mathbf{r}_{i}, t\right)$, $k_{2}^{(N)}\left(\mathbf{r}_{1}, \mathbf{r}_{2}, t\right), \ldots, k_{s}^{(N)}\left(\mathbf{r}_{1}, \mathbf{r}_{2}, \ldots \mathbf{r}_{s}, t\right)$ remain uniquely prescribed by the 1-body PDF, being given by

$$
\begin{array}{r}
k_{1}^{(N)}\left(\mathbf{r}_{1}, t\right) \equiv F_{1}\left\{\prod_{j=2, N} \frac{\rho_{1}^{(N)}\left(\mathbf{x}_{j}, t\right)}{k_{1}^{(N)}\left(\mathbf{r}_{j}, t\right)}\right\}, \\
k_{2}^{(N)}\left(\mathbf{r}_{1}, \mathbf{r}_{2}, t\right) \equiv F_{2}\left\{\prod_{j=s+1, N} \frac{\rho_{1}^{(N)}\left(\mathbf{x}_{j}, t\right)}{k_{1}^{(N)}\left(\mathbf{r}_{j}, t\right)}\right\},
\end{array}
$$

$$
k_{s}^{(N)}\left(\mathbf{r}_{1}, \mathbf{r}_{2}, \ldots \mathbf{r}_{s}, t\right) \equiv F_{s}\left\{\prod_{j=s+1, N} \frac{\rho_{1}^{(N)}\left(\mathbf{x}_{j}, t\right)}{k_{1}^{(N)}\left(\mathbf{r}_{j}, t\right)}\right\}
$$

where $F_{s}$ denotes the integral operator

$$
F_{s} \equiv \int_{\Gamma_{N}} d \overline{\mathbf{x}} \bar{\Theta}^{(N)}(\overline{\mathbf{r}}) \prod_{i=1, s} \delta\left(\mathbf{x}_{i}-\overline{\mathbf{x}}_{i}\right) \text {. }
$$

Therefore, since in the collisionless subset of $\Omega^{(N)}$ the prescriptions (A.11) and (A.13) are equivalent, in the same subset the 1- and 2-body occupation coefficients, written in terms of (A.11), become explicitly

$$
\begin{aligned}
& k_{1}^{(N)}\left(\mathbf{r}_{1}, t\right)=\int_{\Gamma_{1(2)}} d \mathbf{x}_{2} \frac{\rho_{1}^{(N)}\left(\mathbf{x}_{2}, t\right)}{k_{1}^{(N)}\left(\mathbf{r}_{2}, t\right)} \Theta_{2}^{(\partial \Omega)}(\overline{\mathbf{r}}) \\
& . \bar{\Theta}\left(\left|\mathbf{r}_{2}-\mathbf{r}_{1}\right|-\sigma\right) \times \int_{\Gamma_{1(3)}} d \mathbf{x}_{3} \frac{\rho_{1}^{(N)}\left(\mathbf{x}_{3}, t\right)}{k_{1}^{(N)}\left(\mathbf{r}_{3}, t\right)} \Theta_{3}^{(\partial \Omega)}(\overline{\mathbf{r}}) \\
& \quad \cdot \prod_{j=1,2} \bar{\Theta}\left(\left|\mathbf{r}_{3}-\mathbf{r}_{j}\right|-\sigma\right) \\
& \ldots \int_{\Gamma_{1(N)}} d \mathbf{x}_{N} \frac{\rho_{1}^{(N)}\left(\mathbf{x}_{N}, t\right)}{k_{1}^{(N)}\left(\mathbf{r}_{N}, t\right)} \Theta_{N}^{(\partial \Omega)}(\overline{\mathbf{r}}) \\
& \quad \cdot \prod_{j=1, N-1} \bar{\Theta}\left(\left|\mathbf{r}_{N}-\mathbf{r}_{j}\right|-\sigma\right),
\end{aligned}
$$

and

$$
\begin{aligned}
& k_{2}^{(N)}\left(\mathbf{r}_{1}, \mathbf{r}_{2}, t\right)=\int_{\Gamma_{1(3)}} d \mathbf{x}_{3} \frac{\rho_{1}^{(N)}\left(\mathbf{x}_{3}, t\right)}{k_{1}^{(N)}\left(\mathbf{r}_{3}, t\right)} \bar{\Theta}_{3}^{(\partial \Omega)}(\overline{\mathbf{r}}) \\
& \cdot \prod_{j=1,2} \bar{\Theta}\left(\left|\mathbf{r}_{3}-\mathbf{r}_{j}\right|-\sigma\right) \\
& \quad \times \int_{\Gamma_{1(4)}} d \mathbf{x}_{4} \frac{\rho_{1}^{(N)}\left(\mathbf{x}_{4}, t\right)}{k_{1}^{(N)}\left(\mathbf{r}_{4}, t\right)} \Theta_{4}^{(\partial \Omega)}(\overline{\mathbf{r}}) \\
& \cdot \prod_{j=1,3} \bar{\Theta}\left(\left|\mathbf{r}_{4}-\mathbf{r}_{j}\right|-\sigma\right) \\
& \ldots \int_{\Gamma_{1(N)}} d \mathbf{x}_{N} \frac{\rho_{1}^{(N)}\left(\mathbf{x}_{N}, t\right)}{k_{1}^{(N)}\left(\mathbf{r}_{N}, t\right)} \Theta_{N}^{(\partial \Omega)}(\overline{\mathbf{r}}) \\
& \cdot \prod_{j=1, N-1} \bar{\Theta}\left(\left|\mathbf{r}_{N}-\mathbf{r}_{j}\right|-\sigma\right) .
\end{aligned}
$$

Accordingly letting $\mathbf{n}_{j j}=\mathbf{r}_{u i j} /\left|\mathbf{r}_{i j}\right|$ with $\mathbf{r}_{i j}=\mathbf{r}_{i}-\mathbf{r}_{j}$, one notices that in the collisionless subset of $\Omega^{(N)}$ the following differential identities hold for all $s=1, N-1$ :

$$
\begin{gathered}
\frac{\partial}{\partial \mathbf{r}_{1}} k_{1}^{(N)}\left(\mathbf{r}_{1}, t\right)=(N-1) \int_{\Gamma_{1(2)}} d \mathbf{x}_{2} \mathbf{n}_{12} \delta \\
\cdot\left(\left|\mathbf{r}_{2}-\mathbf{r}_{1}\right|-\sigma\right) \times k_{2}^{(N)}\left(\mathbf{r}_{1}, \mathbf{r}_{2}, t\right) \bar{\Theta}_{2}^{(\partial \Omega)}(\overline{\mathbf{r}})
\end{gathered}
$$




$$
\begin{aligned}
& \cdot \widehat{\rho}_{1}^{(N)}\left(\mathbf{x}_{2}, t\right), \\
& \frac{\partial}{\partial \mathbf{r}_{1}} k_{2}^{(N)}\left(\mathbf{r}_{1}, \mathbf{r}_{2}, t\right)=(N-2) \\
& \cdot \int_{\Gamma_{1(3)}} d \mathbf{x}_{3} \mathbf{n}_{13} \delta\left(\left|\mathbf{r}_{3}-\mathbf{r}_{1}\right|-\sigma\right) \\
& \times \prod_{j=1,2 ; j \neq 1} \bar{\Theta}\left(\left|\mathbf{r}_{3}-\mathbf{r}_{j}\right|-\sigma\right) \bar{\Theta}_{3}^{(\partial \Omega)}(\overline{\mathbf{r}}) \\
& \cdot k_{3}^{(N)}\left(\mathbf{r}_{1}, \mathbf{r}_{2}, \mathbf{r}_{3}, t\right) \widehat{\rho}_{1}^{(N)}\left(\mathbf{x}_{3}, t\right), \\
& \frac{\partial}{\partial \mathbf{r}_{2}} k_{2}^{(N)}\left(\mathbf{r}_{1}, \mathbf{r}_{2}, t\right)=(N-2) \int_{\Gamma_{1(3)}} d \mathbf{x}_{3} \mathbf{n}_{23} \delta \\
& \cdot\left(\left|\mathbf{r}_{3}-\mathbf{r}_{2}\right|-\sigma\right) \times \prod_{j=1,2 ; j \neq 2} \bar{\Theta}\left(\left|\mathbf{r}_{3}-\mathbf{r}_{j}\right|-\sigma\right) \bar{\Theta}_{3}^{(\partial \Omega)} \\
& \cdot(\overline{\mathbf{r}}) k_{3}^{(N)}\left(\mathbf{r}_{1}, \mathbf{r}_{2}, \mathbf{r}_{3}, t\right) \hat{\rho}_{1}^{(N)}\left(\mathbf{x}_{3}, t\right), \\
& \text {... } \\
& \frac{\partial}{\partial \mathbf{r}_{1}} k_{s}^{(N)}\left(\mathbf{r}_{1}, \mathbf{r}_{2}, \ldots, \mathbf{r}_{s}, t\right)=(N-s) \int_{\Gamma_{1(s+1)}} d \mathbf{x}_{s+1} \mathbf{n}_{1 s+1} \\
& \cdot \delta\left(\left|\mathbf{r}_{s+1}-\mathbf{r}_{1}\right|-\sigma\right) \times \prod_{j=1, s ; j \neq 1} \bar{\Theta}\left(\left|\mathbf{r}_{s+1}-\mathbf{r}_{j}\right|-\sigma\right) \\
& \cdot \bar{\Theta}_{3}^{(\partial \Omega)}(\overline{\mathbf{r}}) k_{s+1}^{(N)}\left(\mathbf{r}_{1}, \mathbf{r}_{2}, \ldots, \mathbf{r}_{s+1}, t\right) \widehat{\rho}_{1}^{(N)}\left(\mathbf{x}_{s+1}, t\right) \\
& \frac{\partial}{\partial \mathbf{r}_{2}} k_{s}^{(N)}\left(\mathbf{r}_{1}, \mathbf{r}_{2}, \ldots, \mathbf{r}_{s}, t\right)=(N-s) \int_{\Gamma_{1(2)}} d \mathbf{x}_{s+1} \mathbf{n}_{2 s+1} \delta \\
& \cdot\left(\left|\mathbf{r}_{s+1}-\mathbf{r}_{2}\right|-\sigma\right) \\
& \times \prod_{j=1, s ; j \neq 2} \bar{\Theta}\left(\left|\mathbf{r}_{s+1}-\mathbf{r}_{j}\right|-\sigma\right) \bar{\Theta}_{3}^{(\partial \Omega)}(\overline{\mathbf{r}}) \\
& \cdot k_{s+1}^{(N)}\left(\mathbf{r}_{1}, \mathbf{r}_{2}, \ldots, \mathbf{r}_{s+1}, t\right) \hat{\rho}_{1}^{(N)}\left(\mathbf{x}_{s+1}, t\right) \\
& \text {... } \\
& \frac{\partial}{\partial \mathbf{r}_{s}} k_{s}^{(N)}\left(\mathbf{r}_{1}, \mathbf{r}_{2}, \ldots, \mathbf{r}_{s}, t\right)=(N-s) \\
& \cdot \int_{\Gamma_{1(s+1)}} d \mathbf{x}_{s+1} \mathbf{n}_{s s+1} \delta\left(\left|\mathbf{r}_{s+1}-\mathbf{r}_{s}\right|-\sigma\right) \\
& \times \prod_{j=1, s ; j \neq s} \bar{\Theta}\left(\left|\mathbf{r}_{s+1}-\mathbf{r}_{j}\right|-\sigma\right) \bar{\Theta}_{3}^{(\partial \Omega)}(\overline{\mathbf{r}}) \\
& \cdot k_{s+1}^{(N)}\left(\mathbf{r}_{1}, \mathbf{r}_{2}, \ldots, \mathbf{r}_{s+1}, t\right) \widehat{\rho}_{1}^{(N)}\left(\mathbf{x}_{s+1}, t\right)
\end{aligned}
$$

As a consequence the following identities (the first one needed to evaluate the rhs of (A.2) in Appendix A)

$$
\begin{aligned}
& L_{1(1)} k_{1}^{(N)}\left(\mathbf{r}_{1}, t\right)=(N-1) \int_{\Gamma_{1(2)}} d \mathbf{x}_{2} \mathbf{v}_{21} \\
& \cdot \mathbf{n}_{21} \delta\left(\left|\mathbf{r}_{2}-\mathbf{r}_{1}\right|-\sigma\right) \bar{\Theta}^{*}\left(\mathbf{r}_{2}\right) k_{2}^{(N)}\left(\mathbf{r}_{1}, \mathbf{r}_{2}, t\right) \\
& \cdot \hat{\rho}_{1}^{(N)}\left(\mathbf{x}_{2}, t\right),
\end{aligned}
$$

$$
\begin{gathered}
\frac{\partial^{2} k_{1}^{(N)}\left(\mathbf{r}_{1}, t\right)}{\partial \mathbf{r}_{1} \cdot \partial \mathbf{r}_{1}}=-(N-1) \int_{\Gamma_{1(2)}} d \mathbf{x}_{2} k_{2}^{(N)}\left(\mathbf{r}_{1}, \mathbf{r}_{2}, t\right) \\
\cdot \delta\left(\left|\mathbf{r}_{2}-\mathbf{r}_{1}\right|-\sigma\right) \bar{\Theta}_{2}^{(\partial \Omega)}(\overline{\mathbf{r}}) \mathbf{n}_{21} \cdot \frac{\partial}{\partial \mathbf{r}_{2}} \widehat{\rho}_{1}^{(N)}\left(\mathbf{x}_{2}, t\right),
\end{gathered}
$$

hold too. However, the alternative realization of the factor $\bar{\Theta}_{i}(\overline{\mathbf{r}})$ given by (A.13) (see Appendix A) has the virtue of excluding explicitly multiple collisions. The consequence is that when such a definition is adopted the differential identities

$$
\begin{aligned}
& \delta\left(\left|\mathbf{r}_{2}-\mathbf{r}_{1}\right|-\sigma\right) \frac{\partial}{\partial \mathbf{r}_{1}} k_{2}^{(N)}\left(\mathbf{r}_{1}, \mathbf{r}_{2}, t\right)=0 \\
& \delta\left(\left|\mathbf{r}_{2}-\mathbf{r}_{1}\right|-\sigma\right) \frac{\partial}{\partial \mathbf{r}_{2}} k_{2}^{(N)}\left(\mathbf{r}_{1}, \mathbf{r}_{2}, t\right)=0
\end{aligned}
$$

both hold identically. The latter equations, in fact, manifestly hold also in the collision subset where $\delta\left(\left|\mathbf{r}_{2}-\mathbf{r}_{1}\right|-\sigma\right) \neq 0$.

\section{Causal and Anticausal Forms of Collisional Boundary Conditions}

For definiteness, let us denote, respectively, the outgoing and incoming $N$-body PDFs $\rho^{(-)(N)}\left(\mathbf{x}^{(-)}\left(t_{i}\right), t_{i}\right)$ and $\rho^{(+)(N)}\left(\mathbf{x}^{(+)}\left(t_{i}\right), t_{i}\right)$, with $\rho^{( \pm)(N)}\left(\mathbf{x}^{( \pm)}\left(t_{i}\right), t_{i}\right)=$ $\lim _{t \rightarrow t_{i}^{( \pm)}} \rho^{(N)}(\mathbf{x}(t), t)$, where $\mathbf{x}^{(-)}\left(t_{i}\right)$ and $\mathbf{x}^{(+)}\left(t_{i}\right)$, with $\mathbf{x}^{( \pm)}\left(t_{i}\right)$ $=\lim _{t \rightarrow t_{i}^{( \pm)}} \mathbf{x}(t)$, are the incoming and outgoing Lagrangian $N$-body states, their mutual relationship being again determined by the collision laws holding for the $S_{N}-$ CDS. Here it is understood that

(i) the $S_{N}$-CDS is referred to a reference frame $O(\mathbf{r}, \tau \equiv$ $\left.t-t_{o}\right)$, having, respectively, spatial and time origins at the point $O$ which belongs to the Euclidean space $\mathbb{R}^{3}$ and at time $t_{o} \in I$;

(ii) in addition, by assumption the time axis is oriented. Such an orientation is referred to as microscopic arrow of time.

For an arbitrary $N$-body $\operatorname{PDF} \rho^{(N)}(\mathbf{x}, t)$ belonging to the extended functional setting and an arbitrary collision event occurring at time $t_{i}$ two possible realizations of the MCBC can in principle be given, both yielding a relationship between the PDFs $\rho^{(+)(N)}$ and $\rho^{(-)(N)}$. In the context of the ab initio statistical approach based on the Master kinetic equation [1-3] these are provided by the two possible realizations of the so-called modified $C B C$ (MCBC). When expressed in Lagrangian form they are realized, respectively, by either the causal and anticausal MCBC, namely,

$$
\rho^{(+)(N)}\left(\mathbf{x}^{(+)}\left(t_{i}\right), t_{i}\right)=\rho^{(-)(N)}\left(\mathbf{x}^{(+)}\left(t_{i}\right), t_{i}\right),
$$

or

$$
\rho^{(-)(N)}\left(\mathbf{x}^{(-)}\left(t_{i}\right), t_{i}\right)=\rho^{(+)(N)}\left(\mathbf{x}^{(-)}\left(t_{i}\right), t_{i}\right) .
$$


The corresponding Eulerian forms of the MCBC can easily be determined (see [2]). The one corresponding to (C.1) is, for example, provided by the condition

$$
\rho^{(+)(N)}\left(\mathbf{x}^{(+)}, t\right)=\rho^{(-)(N)}\left(\mathbf{x}^{(+)}, t\right),
$$

where now $\mathbf{x}^{(+)}$denotes again an arbitrary outgoing collision state.

Once the time axis is oriented, i.e., the microscopic arrow of time is prescribed, the validity of the causality principle in the reference frame $\left(\mathbf{r}, \tau \equiv t-t_{o}\right)$ manifestly requires invoking (C.1). Indeed, (C.1) predicts the future (i.e., outgoing) PDF from the past (incoming) one. Therefore the choice (C.1) is the one which is manifestly consistent with the causality principle. On the other hand, if the arrow of time is changed, i.e., the time-reversal transformation with respect to the initial time (or time-origin) $t_{o}$, i.e., the map between the two reference frames

$$
O\left(\mathbf{r}, \tau \equiv t-t_{o}\right) \longrightarrow O\left(\mathbf{r}, \tau^{\prime}\right)
$$

with $\tau^{\prime}=-\tau$ is performed, it is obvious that for the transformed reference frame $O\left(\mathbf{r}, \tau^{\prime}\right)$ the form of $\mathrm{CBC}$ consistent with causality principle becomes that given by (C.2). Analogous conclusions hold if a velocity-reversal is performed, implying the incoming states and corresponding PDF must be exchanged with corresponding outgoing ones and vice versa.

\section{Treatment of Case $N=2$}

For completeness let us briefly comment on the particular realization of MPI/DKE theory which is achieved in the special case $N=2$. For this purpose, one notices that-thanks to (B.2) recalled in Appendix B (see also [3]) - in this case by construction $k_{2}^{(N)}\left(\mathbf{r}_{1}, \mathbf{r}_{2}, t\right)$ simply reduces to

$$
k_{2}^{(N)}\left(\mathbf{r}_{1}, \mathbf{r}_{2}, t\right) \equiv 1 \text {. }
$$

Accordingly, once the same prescription is invoked, both the Master kinetic equation (A.4) and the corresponding Master collision operator (A.5) remain formally unchanged. In a similar way it is important to remark that the expression of the functional $(\partial / \partial t) K_{M}\left(\rho_{1}^{(N)}(t), \mathbf{b}\right) \equiv W_{M}\left(\rho_{1}^{(N)}(t), \mathbf{b}\right)$ given by (53) is still correct also in such a case, being now given by

$$
\begin{aligned}
\frac{\partial}{\partial t} K_{M} & \left(\rho_{1}^{(N)}(t), \mathbf{b}\right) \equiv W_{M}\left(\rho_{1}^{(N)}(t), \mathbf{b}\right) \\
= & -(N-1) \sigma^{2} \int_{U_{1(1)}} d \mathbf{v}_{1} \int_{U_{1(2)}} d \mathbf{v}_{2} \\
& \times \int_{\Omega} d \mathbf{r}_{1} \int^{(-)} d \mathbf{\Sigma}_{21} \frac{\partial \widehat{\rho}_{1}^{(N)}\left(\mathbf{r}_{1}, \mathbf{v}_{1}^{(+)}, t\right)}{\partial \mathbf{r}_{1}} \\
& \cdot \frac{\partial \widehat{\rho}_{1}^{(N)}\left(\mathbf{r}_{2}=\mathbf{r}_{1}+\sigma \mathbf{n}_{21}, \mathbf{v}_{2}^{(+)} t\right)}{\partial \mathbf{r}_{2}} \\
& \times\left|\mathbf{v}_{12}^{(+)} \cdot \mathbf{n}_{12}\right|\left(\mathbf{b} \cdot \mathbf{n}_{12}\right)^{2}\left(\mathbf{n}_{12} \cdot \mathbf{v}_{12}^{(+)}\right)^{2} \leq 0 .
\end{aligned}
$$

It is then immediate to infer the validity of both the PMI theorem (Theorem 2) and the DKE property for the Master kinetic equation (Theorem 3). As a consequence one concludes that MPI/DKE theory holds also in the special case $N=2$. This conclusion is not unexpected. In fact, binary collisions, as indicated above, are responsible for the MPI/DKE phenomenology and in such a case can only occur between particles 1 and 2 .

\section{Data Availability}

No availability statement is needed.

\section{Conflicts of Interest}

The authors declare that they have no conflicts of interest.

\section{Acknowledgments}

This work is dedicated to the dearest memory of Flavia, wife of Massimo Tessarotto, recently passed away. The investigation was developed in part within the research projects: (A) the Albert Einstein Center for Gravitation and Astrophysics, Czech Science Foundation no. 14-37086G; (B) the research projects of the Czech Science Foundation GAČR Grant no. 14-07753P; (C) the Grant no. 02494/2013/RRC “Kinetický prístup $k$ proudĕní tekutin" (Kinetic approach to fluid flow) in the framework of the "Research and Development Support in Moravian-Silesian Region", Czech Republic. Initial framework and motivations of the investigation were based on the research projects developed by the Consortium for Magnetofluid Dynamics (University of Trieste, Italy) and the MIUR (Italian Ministry for Universities and Research) PRIN Research Program "Problemi Matematici delle Teorie Cinetiche e Applicazioni" (Mathematical Problems of Kinetic Theories and Applications), University of Trieste, Italy. One of the authors (Massimo Tessarotto) is grateful to the International Center for Theoretical Physics (Miramare, Trieste, Italy) for the hospitality during the preparation of the manuscript.

\section{References}

[1] M. Tessarotto, C. Cremaschini, and M. Tessarotto, "On the conditions of validity of the Boltzmann equation and Boltzmann H-theorem," The European Physical Journal Plus, vol. 128, no. 3, 2013.

[2] M. Tessarotto and C. Cremaschini, "First-principle proof of the modified collision boundary conditions for the hard-sphere system," Physics Letters A, vol. 378, no. 26-27, pp. 1760-1766, 2014.

[3] Tessarotto M. and C. Cremaschini, The European Physical Journal Plus, vol. 129, 2014.

[4] M. Tessarotto, C. Cremaschini, M. Mond, C. Asci, A. Soranzo, and G. Tironi, "On the Boltzmann-Grad limit for smooth hardsphere systems," Foundations of Physics. An International Journal Devoted to the Conceptual Bases and Fundamental Theories of Modern Physics, vol. 48, no. 3, pp. 271-294, 2018.

[5] R. Clemente and M. Tessarotto, "On the derivation of the Boltzmann equation from the BBGKY hierarchy," Transport Theory 
and Statistical Physics. An International Journal for Rapid Communication, vol. 8, no. 1, pp. 1-16, 1979.

[6] C. Cercignani, Mathematical Methods in Kinetic Theory, Plenum Press, New York, NY, USA, 1969.

[7] S. G. Brush, Weitere Studien über das W ärmegleichgewicht unter Gasmolekülen, Wiener Berichte, 66, 275-370, in WA I, paper 23, 1872.

[8] D. Enskog, "Kungl. Svensk Vetenskps Akademiens," English translation by S. G. Brush, vol. 4, 1921.

[9] S. Chapman and T. G. Cowling, The Mathematical Theory of Nonuniform Gases, Cambridge University Press, 1951.

[10] H. Grad, "Thermodynamics of gases," Handbook der Physik, vol. 205, 1958.

[11] C. Cercignani, Theory And Application of The Boltzmann Equation, Scottish Academic Press, Edinburgh and London, 1975.

[12] C. Cercignani, The Boltzmann Equation and Its Applications, vol. 67 of Applied Mathematical Sciences, Springer, New York, NY. USA, 1988.

[13] M. Tessarotto, C. Cremaschini, C. Asci, A. Soranzo, and G. Tironi, "Global validity of the Master kinetic equation for hardsphere systems," The European Physical Journal Plus, vol. 130, no. 8, 2015.

[14] Y. G. Sinai, "Dynamical systems with elastic reflections," Russian Mathematical Surveys, vol. 25, p. 137, 1970.

[15] Y. G. Sinai, "Dynamical Systems II," in Ergodic Theory with Applications to Dynamical Systems and Statistical Mechanics, Springer-Verlag, Berlin, Germany, 1989.

[16] M. Tessarotto and M. Ellero, "Unique representation of an inverse-kinetic theory for incompressible Newtonian fluids," in Proceedings of the AIP Conference, vol. 762, p. 108, 2005.

[17] C. Cremaschini and M. Tessarotto, "Theory of spatially nonsymmetric kinetic equilibria for collisionless plasmas," Physics of Plasmas, vol. 20, no. 1, 2013.

[18] C. Cremaschini, Z. Stuchlík, and M. Tessarotto, "Kinetic theory of quasi-stationary collisionless axisymmetric plasmas in the presence of strong rotation phenomena," Physics of Plasmas, vol. 20, no. 5, 2013.

[19] C. Cremaschini, M. Tessarotto, and Z. Stuchlík, "Kinetic equilibria of relativistic collisionless plasmas in the presence of nonstationary electromagnetic fields," Physics of Plasmas, vol. 21, no. 3, 2014.

[20] O. E. Lanford, "Time evolution of large classical systems," in Proceedings of Dynamical Systems, Theory and Applications, 1974 Battelle Rencontre on Dynamical Systems, J. Moser, Ed., Lecture Notes in Physics, Vol. 38, Springer,Berlin, p. 1, 1975.

[21] O. E. Lanford and Soc., "Société mathématique de France," Astérisque, vol. 40, p. 117, 1976.

[22] O. E. Lanford, "The hard sphere gas in the Boltzmann-Grad limit," Physica A: Statistical Mechanics and its Applications, vol. 106, no. 1-2, pp. 70-76, 1981.

[23] C. Villani, "Entropy production and convergence to equilibrium for the Boltzmann equation," in Proceedings of the XIVth International Congress on Mathematical Physics, pp. 130-144, Lisbon, Portugal, 2006.

[24] J. Uffink and G. Valente, "Lanford's theorem and the emergence of irreversibility," Foundations of Physics. An International Journal Devoted to the Conceptual Bases and Fundamental Theories of Modern Physics, vol. 45, no. 4, pp. 404-438, 2015.

[25] V. Ardourel, "Irreversibility in the derivation of the Boltzmann equation," Foundations of Physics. An International Journal
Devoted to the Conceptual Bases and Fundamental Theories of Modern Physics, vol. 47, no. 4, pp. 471-489, 2017.

[26] A. Drory, "Is there a reversibility paradox? Recentering the debate on the thermodynamic time arrow," Studies in History and Philosophy of Science. Part B. Studies in History and Philosophy of Modern Physics, vol. 39, no. 4, pp. 889-913, 2008.

[27] J. Loschmidt, Akademie der Wissenschaften zu Wien, vol. 73, p. $128,1876$.

[28] L. Boltzmann, Wissenschaftliche Abhandlungen, 275, 1877, in Boltzmann Vol. II, pp. 112-148, 1909.

[29] P. Ehrenfest and T. Ehrenfest, The Conceptual Foundations of The Statistical Approach in Mechanics, Cornell University Press, New York, NY, USA, 1912.

[30] C. Cercignani, " $H$-theorem and trend to equilibrium in the kinetic theory of gases," Polish Academy of Sciences. Institute of Fundamental Technological Research. Archives of Mechanics (Archiwum Mechaniki Stosowanej), vol. 34, no. 3, pp. 231-241 (1983), 1982.

[31] J. L. Lebowitz and Phys. Today, Physics Today (Letters Section), p. 115, 1994.

[32] G. Gallavotti, Nonequilibrium and irreversibility, Theoretical and Mathematical Physics, Springer, 2014.

[33] M. Tessarotto, M. Mond, and C. Asci, "MOND as the weak field limit of an extended metric theory of gravity with torsion," The European Physical Journal Plus, vol. 213, p. 132, 2017. 


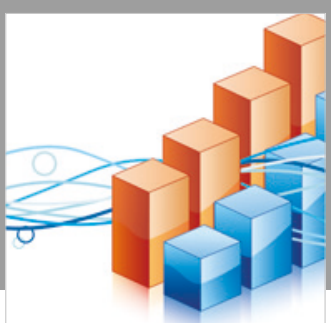

Advances in

Operations Research

\section{-n-m}
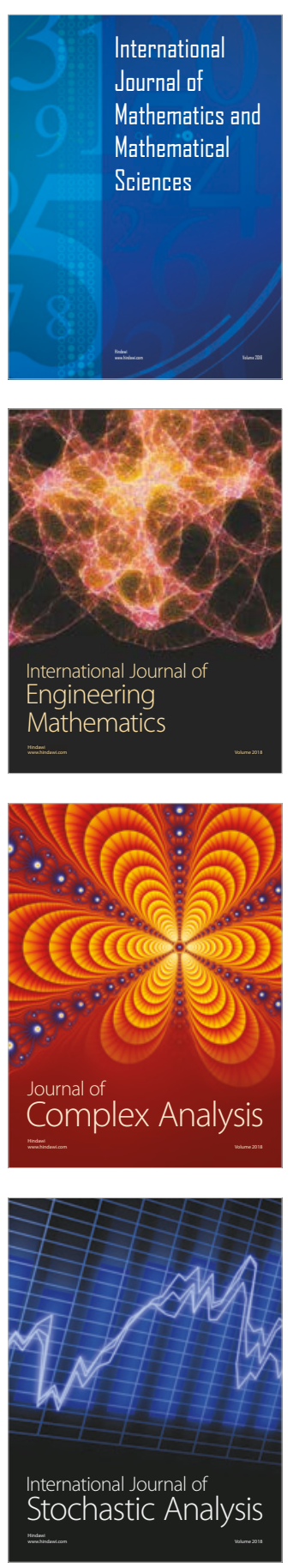
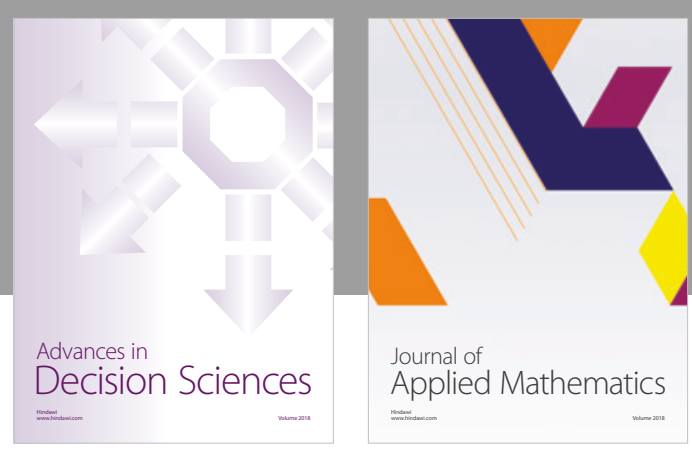

Journal of

Applied Mathematics
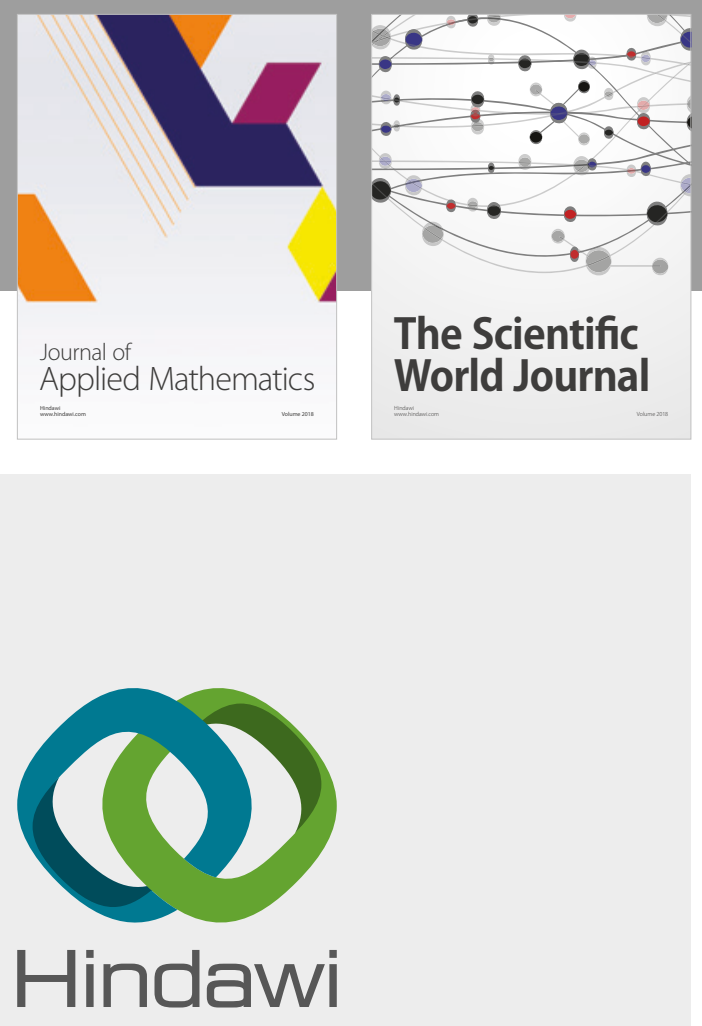

Submit your manuscripts at

www.hindawi.com

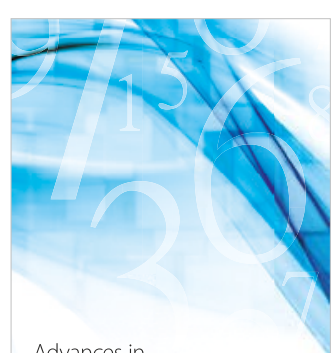

Advances in
Numerical Analysis
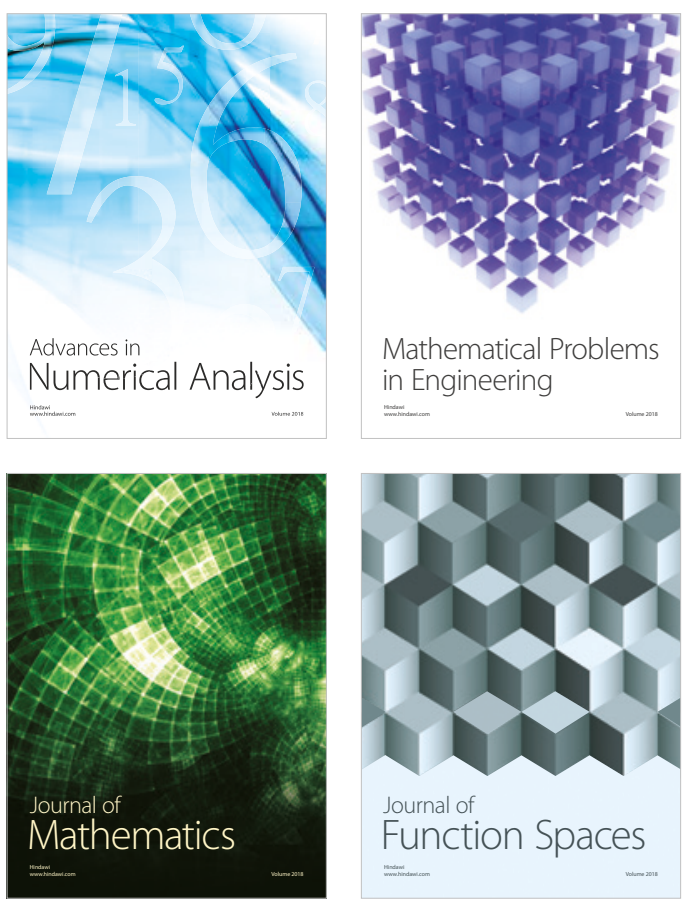

Mathematical Problems in Engineering

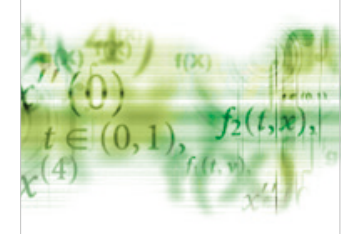

International Journal of

Differential Equations

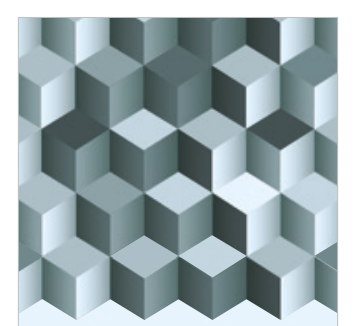

Journal of

Function Spaces

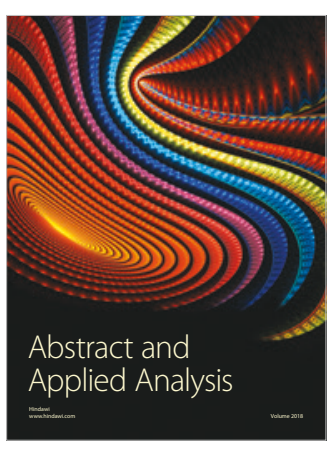

The Scientific

World Journal

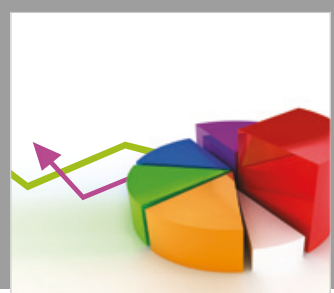

Journal of

Probability and Statistics
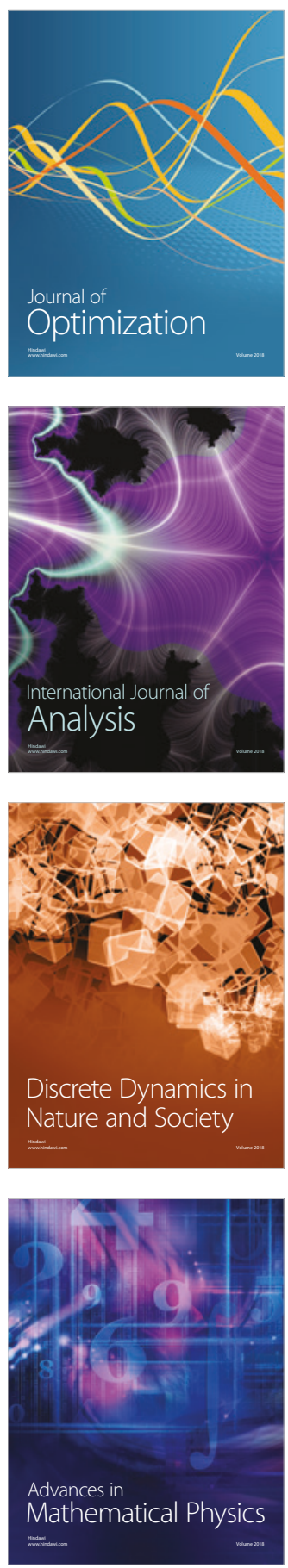